\title{
Exploring the micro-mechanics of triaxial instability in granular materials
}

\section{J. C. LOPERA PEREZ*, C. Y. KWOK*, C. O’SULLIVAN†, X. HUANG ${ }^{*}$ and K. J. HANLEY§}

\begin{abstract}
The instability of granular materials due to water infiltration under fully drained conditions has been previously considered in experimental studies. While laboratory experiments can provide macro-scale insight into drained instability, the micro-mechanics under such conditions are yet to be explored. This study has employed the discrete-element method (DEM) to simulate constant shear drained (CSD) tests for an ideal soil. CSD tests were initiated from a range of packing densities and stress conditions. The DEM simulations were able to qualitatively replicate laboratory CSD tests. The choice of the loading control parameter was seen to play a central role in the macro-scale second-order work to identify an effective failure. All samples considered attained an onset of instability that coincided with fluctuations in the second-order work from a particle scale. The time of occurrence of the onset of instability was seen to depend on initial packing density and stress state. A change in the evolution of macro- and micro-mechanical quantities, showing either a sharp increase or decrease, was observed once the CSD conditions had been reached. Finally, conventional drained then constant volume (CDCV) tests were carried out where the appearance of instabilities and the evolution of macro and micro quantities were found to be different from those observed in CSD tests. The results presented in this study indicate that the constant shear drained loading conditions can result in more unfavourable situations than for the undrained loading condition.
\end{abstract}

KEYWORDS: discrete-element modelling; fabric/structure of soils; particle-scale behaviour

\section{INTRODUCTION}

The possibility of collapse of a granular soil under static and fully drained conditions was first noticed by Lindenberg \& Koning (1981). Later it was shown that water infiltration under saturated drained conditions could trigger the failure of geotechnical structures, such as slopes, which could also be the cause of debris flow (Eckersley, 1990; Olson et al., 2000). In order to investigate this kind of instability, stresscontrolled constant shear drained (CSD) triaxial tests have been employed to mimic the infiltration of water and the stress state in a slope (e.g. Sasitharan et al., 1993).

In CSD tests, instability is considered to be the rapid development of large plastic strains as the soil becomes unable to sustain the imposed stress (Sawicki \& Swidzinski, 2010; Chu et al., 2012). The onset of instability under drained conditions coincides with an increase of strain rate, developing large strains that cause the sample to become uncontrollable (Nova, 1994). Most of the research that has been carried out in this subject consists of laboratory tests (Sasitharan et al., 1993; Anderson \& Riemer, 1995; Zhu \& Anderson, 1998; Gajo et al., 2000; Chu et al., 2003, 2012). Several constitutive models that can successfully simulate the instability

Manuscript received 14 September 2015; revised manuscript accepted 1 April 2016. Published online ahead of print 6 May 2016. Discussion on this paper closes on 1 February 2017, for further details see p. ii.

* Department of Civil Engineering, The University of Hong Kong, Hong Kong.

$\dagger$ Department of Civil and Environmental Engineering, Imperial College London, London, UK.

+ Department of Geotechnical Engineering, Tongji University, Shanghai, China.

$\S$ Institute for Infrastructure and Environment, School of Engineering, The University of Edinburgh, Edinburgh, Scotland, UK. observed in CSD tests have also been proposed (Darve et al., 2004; Sawicki \& Swidzinski, 2010; Ramos et al., 2012).

Hill's condition of stability (Hill, 1958) has been proven to be useful for identifying the onset of instability in CSD tests (Darve et al., 2004, 2007; Sibille et al., 2007; Daouadji et al., 2010; Nicot et al., 2011; Hadda et al., 2013). Instability and collapse of granular soils under CSD conditions can happen to both loose and dense samples, either in a dry or a saturated state (Skopek et al., 1994; Chu et al., 2003). This differs markedly from undrained conditions, under which instability is mainly observed in saturated loose to medium dense samples. Undrained tests are usually carried out under straincontrolled conditions, where the onset of instability is correlated with an initial peak in deviator stress, according to the concept of collapse surface introduced by Sladen et al. (1985). The relationship between the state parameter (as proposed by Been \& Jefferies (1985)) and the stress ratio at the onset of instability for undrained tests can also serve as an indicator of instability for CSD tests (Chu et al., 2003, 2012). For conventional undrained tests this relationship is bound by a critical density that marks the limit at which samples can attain an unstable behaviour (Lindenberg \& Koning, 1981).

Extensive information from a macro scale has been reported for CSD tests, but the micro-mechanics of the onset of instability and subsequent collapse for these stress conditions are yet to be explored. Moreover, the underlying mechanism that accounts for drained and undrained instabilities remains unclear. Ning et al. (2013) showed that the discrete-element method (DEM) (Cundall \& Strack, 1979) can act as a tool to simulate CSD tests. The aim of the present contribution is to fill these gaps by conducting DEM tests to simulate the instability behaviour of an ideal granular soil.

\section{DISCRETE-ELEMENT METHOD SIMULATIONS}

This study used a modified version of the open-source code LAMMPS (Plimpton, 1995). As shown in Fig. 1, 
three-dimensional numerical samples consisting of 22312 initially non-contacting spherical particles were created as a representative volume element, enclosed within a cuboidal periodic cell to avoid boundary effects (Thornton, 2000; Huang et al., 2014). The stresses in the periodic cell were determined from the stress tensor defined as $\overline{\boldsymbol{\sigma}}_{i j}=1 / V \sum_{1}^{N_{\mathrm{c}}} \boldsymbol{I}_{i}^{\mathrm{c}} f_{j}^{\mathrm{c}}$, where $\overline{\boldsymbol{\sigma}}_{i j}$ is the stress tensor, $V$ is the volume of the periodic cell, $N_{\mathrm{c}}$ is the total number of contacts, and $\boldsymbol{I}_{i}^{\mathrm{c}}$ and $f_{j}^{\mathrm{c}}$ are the branch vector and interparticle contact force corresponding to contact $c$, respectively (Bagi, 1996; Potyondy \& Cundall, 2004). The particle size distribution (PSD) used for all the simulations is representative of

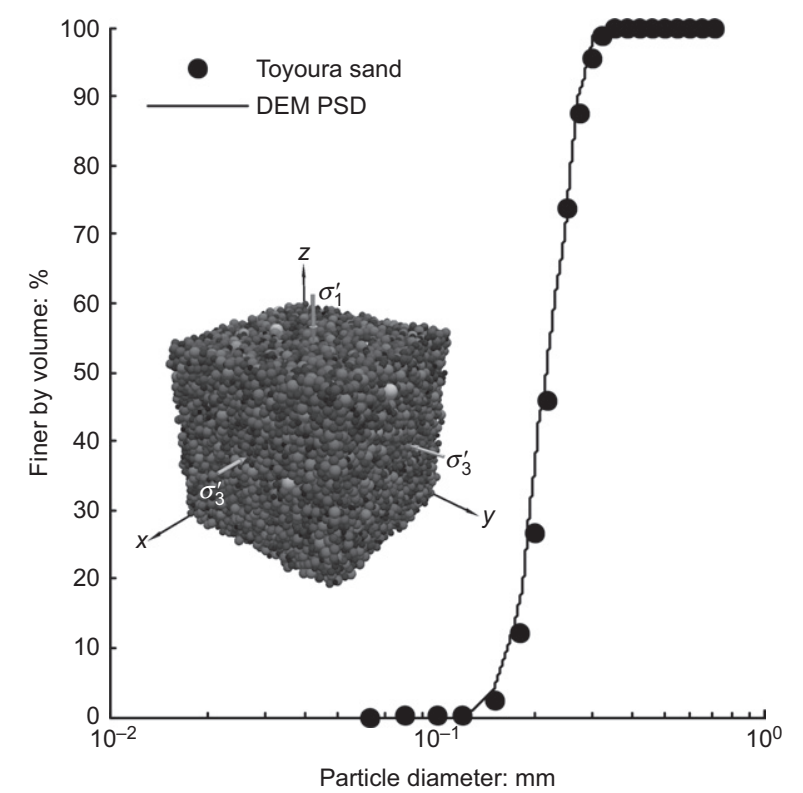

Fig. 1. Particle size distribution of numerical samples compared with laboratory data for Toyoura sand. The inset figure shows a representative sample for simulations following isotropic compression
Toyoura sand (Fig. 1). A simplified Hertz-Mindlin contact model was used. The input parameters were a particle shear modulus $(G)$ of $29 \mathrm{GPa}$, a particle Poisson ratio $(v)$ of $0 \cdot 12$, a particle density $(\rho)$ of $2650 \mathrm{~kg} / \mathrm{m}^{3}$ and a local damping coefficient of $0 \cdot 1$. A parametric study which compared damping coefficients of $0 \cdot 01$ and $0 \cdot 1$ confirmed that this damping value was low enough to have negligible influence on the observed behaviour. Gravity was inactive during these simulations. All simulations were run on a high-performance cluster using a stable time step of $5 \cdot 3 \mathrm{~ns}$. The time step $(\delta t)$ for the analyses was calculated as $0 \cdot 1 \sqrt{m / k}$ where $m / k$ is the minimum ratio of particle mass/contact stiffness for the system.

The periodic cell was initially deformed until the system reached an isotropic stress state with an initial mean effective stress $\left(p_{0}^{\prime}\right)$ of $500 \mathrm{kPa}$. The system was then subjected to numerical cycling until both $p^{\prime}$ and the number of contacts became constant, indicating equilibrium. The void ratio of each sample at the end of isotropic compression was controlled using different inter-particle friction coefficients $(\mu)$ during the isotropic compression stage. Samples with three different initial densities were considered: dense $\left(e_{0}=0.5533\right)$, medium dense $\left(e_{0}=0.6238\right)$ and loose $\left(e_{0}=0.6491\right)$. For all tests, $\mu$ was set to $0 \cdot 25$ after the isotropic compression stage, consistent with values for real quartz particles, where values for $\mu$ are in the range of $0 \cdot 12-0 \cdot 35$, as was observed by Senetakis et al. (2013). The tests carried out included CSD, conventional drained (CD), constant volume (CV) and conventional drained then constant volume (CDCV) tests.

Details of all the tests performed are included in Table 1. In this table, the test notation is divided in four parts, indicating the type of test, $p_{0}^{\prime}, e_{0}$ and (only for CSD and CDCV) the $q$ at which either the CSD or the constant volume conditions, respectively, were initiated. The strain rate used for the $\mathrm{CV}$ and $\mathrm{CD}$ tests was calculated using the inertial number defined as $I=\dot{\varepsilon} d \sqrt{\rho / p^{\prime}}$ where $\dot{\varepsilon}$ is the shear rate, $d$ is the mean particle size of the assembly, $\rho$ is the grain density and $p^{\prime}$ is the mean effective stress. Maintaining $I \leq 2.5 \times 10^{-3}$, ensures quasi-steady conditions during the shearing process (MiDi, 2004; da Cruz et al., 2005; Lopera et al., 2016).

Table 1. List of tests conducted

\begin{tabular}{|c|c|c|c|c|c|c|c|c|}
\hline Test ID & $e_{0}$ & $e_{\mathrm{CSD}}$ & $p_{0}^{\prime}: \mathrm{kPa}$ & $q_{\mathrm{csd}}: \mathrm{kPa}$ & $q_{\mathrm{cv}}: \mathrm{kPa}$ & $e_{\mathrm{cs}}$ & $p_{\mathrm{cs}}^{\prime}: \mathrm{kPa}$ & $q_{\mathrm{cs}}: \mathrm{kPa}$ \\
\hline CSD-500-0.5533-118 & $0 \cdot 5533$ & $0 \cdot 5532$ & 500 & 118 & - & - & - & - \\
\hline CSD-500-0.6238-118 & $0 \cdot 6238$ & $0 \cdot 6236$ & 500 & 118 & - & - & - & - \\
\hline CSD-500-0.6491-118 & $0 \cdot 6491$ & $0 \cdot 6488$ & 500 & 118 & - & - & - & - \\
\hline CSD-500-0.5533-250 & $0 \cdot 5533$ & $0 \cdot 5530$ & 500 & 250 & - & - & - & - \\
\hline CSD-500-0.6238-250 & $0 \cdot 6238$ & $0 \cdot 6234$ & 500 & 250 & - & - & - & - \\
\hline CSD-500-0·6491-250 & $0 \cdot 6491$ & $0 \cdot 6450$ & 500 & 250 & - & - & - & - \\
\hline CDCV-500-0.5533-118 & $0 \cdot 5533$ & $0 \cdot 5532$ & 500 & - & 118 & - & - & - \\
\hline CDCV-500-0.6238-118 & 0.6238 & $0 \cdot 6236$ & 500 & - & 118 & - & - & - \\
\hline CDCV-500-0.6491-118 & $0 \cdot 6491$ & $0 \cdot 6488$ & 500 & - & 118 & - & - & - \\
\hline CV-500-0.6238 & $0 \cdot 6238$ & - & 500 & - & - & $0 \cdot 6238$ & $5176 \cdot 8$ & $3586 \cdot 6$ \\
\hline CV-500-0.6280 & $0 \cdot 6280$ & - & 500 & - & - & $0 \cdot 6280$ & $4064 \cdot 0$ & $2795 \cdot 6$ \\
\hline CV-500-0.6312 & $0 \cdot 6312$ & - & 500 & - & - & $0 \cdot 6312$ & $2775 \cdot 7$ & $1893 \cdot 2$ \\
\hline CV-500-0.6381 & $0 \cdot 6381$ & - & 500 & - & - & $0 \cdot 6381$ & $32 \cdot 11$ & $22 \cdot 10$ \\
\hline CV-500-0.6438 & $0 \cdot 6438$ & - & 500 & - & - & - & - & - \\
\hline CV-500-0.6469 & $0 \cdot 6469$ & - & 500 & - & - & - & - & - \\
\hline CV-500-0.6491 & $0 \cdot 6491$ & - & 500 & - & - & - & - & - \\
\hline CV-500-0.6533 & 0.6533 & - & 500 & - & - & - & - & - \\
\hline CV-500-0.6585 & $0 \cdot 6585$ & - & 500 & - & - & - & - & - \\
\hline CV-500-0.6614 & $0 \cdot 6614$ & - & 500 & - & - & - & - & - \\
\hline CD-100-0.5928 & $0 \cdot 5928$ & - & 100 & - & - & 0.6369 & $128 \cdot 7$ & $86 \cdot 77$ \\
\hline CD-500-0.5533 & 0.5533 & - & 500 & - & - & $0 \cdot 6304$ & $659 \cdot 1$ & $477 \cdot 3$ \\
\hline CD-500-0.6059 & $0 \cdot 6059$ & - & 500 & - & - & 0.6325 & 656.03 & $468 \cdot 06$ \\
\hline CD-500-0.6142 & $0 \cdot 6142$ & - & 500 & - & - & 0.6341 & $1309 \cdot 7$ & $928 \cdot 9$ \\
\hline CD-2500-0.5781 & $0 \cdot 5781$ & - & 2500 & - & - & 0.6303 & $3232 \cdot 7$ & $2197 \cdot 6$ \\
\hline CD $-5000-0 \cdot 6482$ & $0 \cdot 6482$ & - & 5000 & - & - & $0 \cdot 6238$ & $6477 \cdot 7$ & $4432 \cdot 3$ \\
\hline
\end{tabular}


The two different stress paths considered in this study are shown in Fig. 2. In the first case (Fig. 2(a)), following isotropic compression, samples were sheared under stresscontrolled drained conditions at constant lateral stresses. As the deviatoric stress $(q)$ reached either $118 \mathrm{kPa}$ or $250 \mathrm{kPa}$ $\left(q / p^{\prime} \approx 0 \cdot 2\right.$ or $\left.q / p^{\prime} \approx 0 \cdot 4\right)$, the stress path was changed to CSD, which was achieved with a servo-control by simultaneously reducing the major and minor principal stresses, $\sigma_{1}^{\prime}$ and $\sigma_{3}^{\prime}$, respectively, by the same amount. Different stress reduction rates $(1 \mathrm{kPa} / 500$ time steps, $1 \mathrm{kPa} / 5000$ time steps and $1 \mathrm{kPa} / 50000$ time steps) were considered, and similar responses were observed. Therefore, only those results for a reduction rate of $1 \mathrm{kPa} / 5000$ time steps are presented in this paper. The CSD path was continued until the sample lost controllability as defined by Nova (1994). In total, six tests were considered for the first loading path.

In order to find the similarities and differences between the CSD tests and CDCV tests at their different stages, instead of initiating CSD tests upon reaching $q / p^{\prime} \approx 0 \cdot 2$, strain-controlled CV tests were performed, that is, following the second type of loading path presented in Fig. 2(b). The loading path just before the start of the CV tests is the same as for the CSD tests. Therefore, differences between the sample responses under CSD and CV conditions can be attributed solely to the different loading conditions.

A third set of tests was performed, consisting of ten conventional $\mathrm{CV}$ strain-controlled tests, and six $\mathrm{CD}$ tests with constant $\sigma_{3}^{\prime}$ and strain controlled to obtain the critical state lines in both $e-p^{\prime}$ and $q-p^{\prime}$ planes, as well as the stress ratio (at instability state)-state parameter relationship. All these tests used samples that were compressed to an

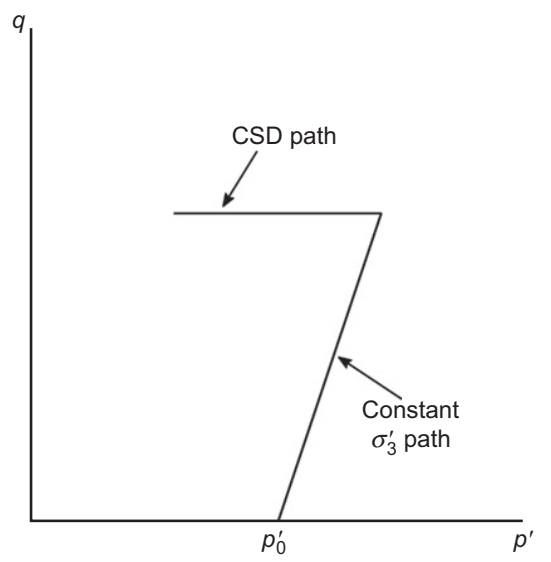

(a)

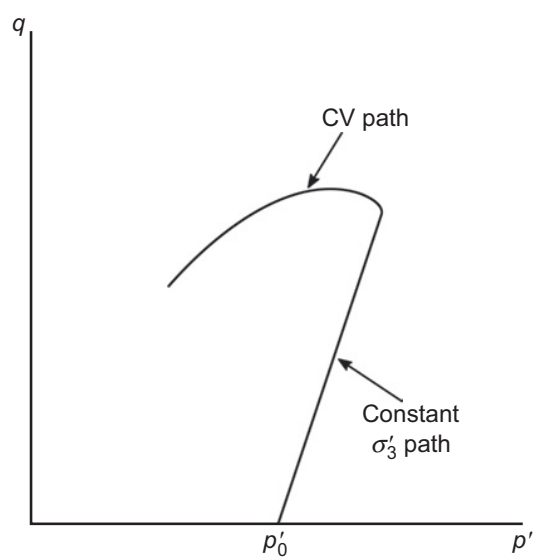

(b)

Fig. 2. Stress paths adopted: (a) drained shearing followed by CSD simulation; (b) drained shearing followed by $\mathrm{CV}$ simulation isotropic confining pressure prior to shearing; all of the $\mathrm{CV}$ tests had an initial $p^{\prime}$ of $500 \mathrm{kPa}$, whereas a range of initial $p^{\prime}$ values between $100 \mathrm{kPa}$ and $5000 \mathrm{kPa}$ was used for the CD tests.

\section{RESULTS}

Stress-deformation response

Figure 3 shows how a constant $\sigma_{3}^{\prime}$ stress path was initially imposed, after which $\sigma_{3}^{\prime}$ was reduced linearly with time under CSD conditions (stress path shown in Fig. 2(a)). Note that in the subsequent figures 'cross' markers indicate the onset of instability for the CSD test, whereas 'plus sign' markers relate the onset of instability for the CDCV test. The instability points were defined by considering the second-order work at the macro and particle scale together with a criterion involving changes in $q$ and $p^{\prime}$, as detailed below. After the onset of instability, even though the servo-control algorithm aims to reduce $\sigma_{1}^{\prime}$ and $\sigma_{3}^{\prime}$ equally, the sample was not able to sustain a constant deviatoric stress.

Results from six CSD tests in the $q-p^{\prime}$ plane are plotted in Fig. 4. Two CSD tests were carried out for each sample, at $q_{\mathrm{CSD}}=118 \mathrm{kPa}$ and $q_{\mathrm{CSD}}=250 \mathrm{kPa}$, where $q_{\mathrm{CSD}}$ denotes the deviatoric stress level at which CSD tests were initiated (void ratios at the onset of the CSD tests are included in Table 1). Circular markers indicate the initiation of the CSD tests for $q_{\mathrm{CSD}}=118 \mathrm{kPa}$, and triangular markers indicate the initiation of the CSD tests for $q_{\mathrm{CSD}}=250 \mathrm{kPa}$. The inset figures

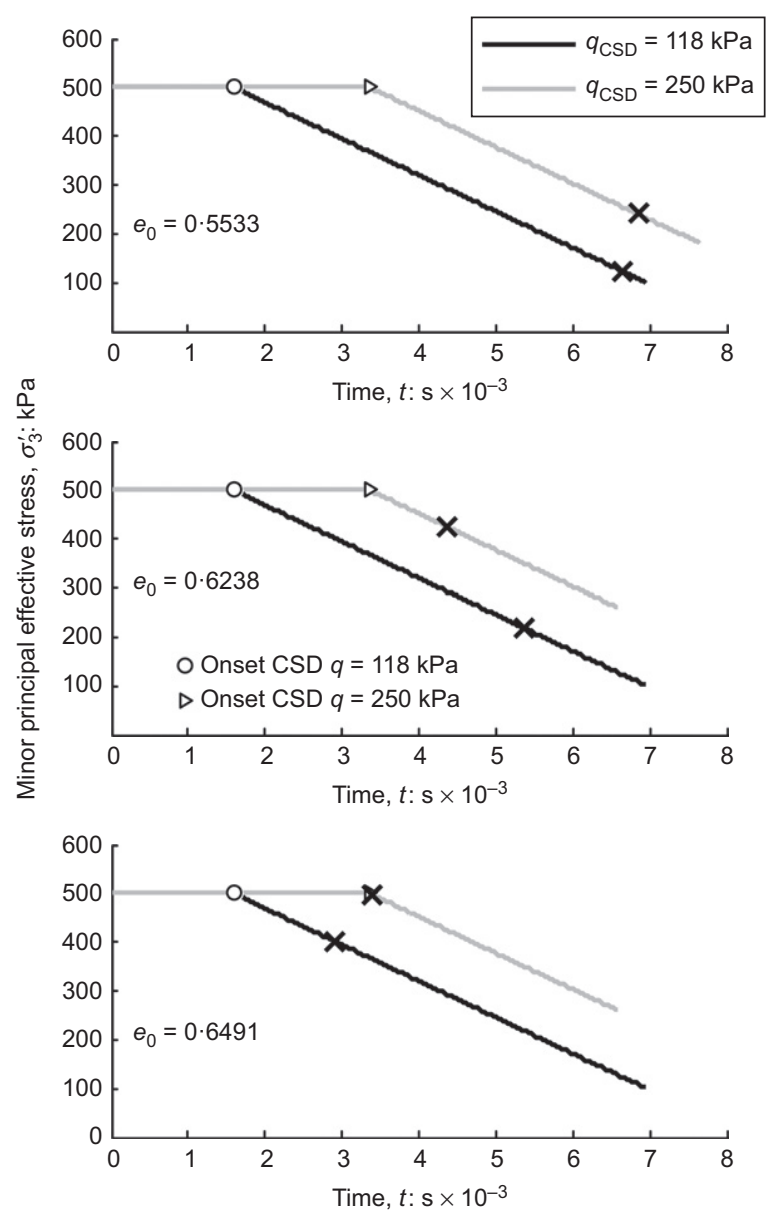

Fig. 3. Minor principal effective stress $\sigma_{3}^{\prime}$ plotted against time. Cross markers indicate the onset of instability for CSD tests. The open circle indicates the onset of CSD conditions for $q_{C S D}=118 \mathrm{kPa}$, while the open triangle indicates the onset of CSD conditions for $q_{\mathrm{CSD}}=250 \mathrm{kPa}$ 


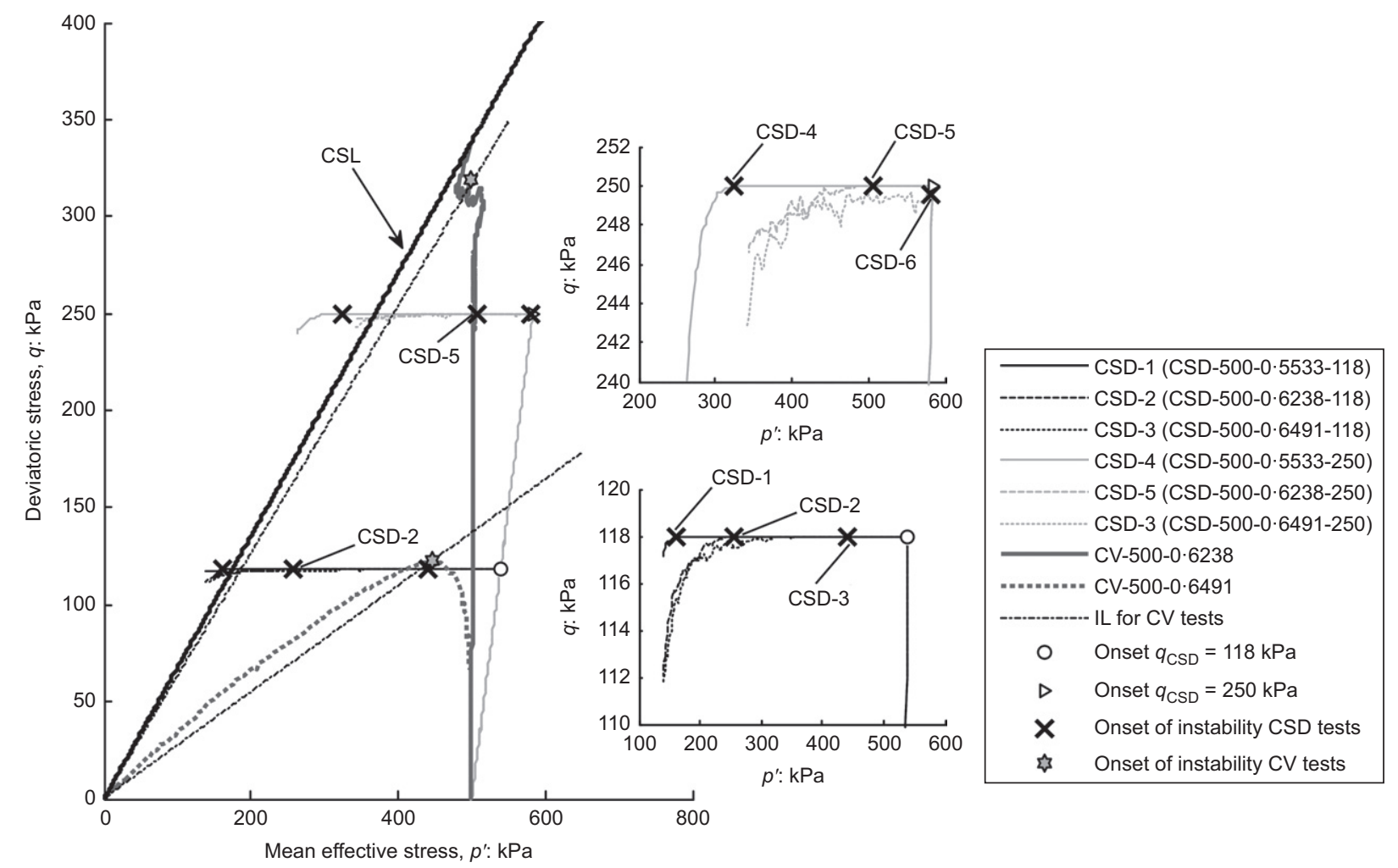

Fig. 4. Deviatoric stress $q$ plotted against mean effective stress $p^{\prime}$ for CSD and CV simulations. Inset figures zoom in to the onset of instability of the CSD tests

provide an enlarged view of the CSD stress paths, where a marked decrease in $q$ is observed that finally leads to a loss in controllability and the collapse of the system as described by Nova (1994). This decrease in $q$ appears at a higher $p^{\prime}$ for looser samples; denser samples follow a longer stress path which crosses the CSL so that decreases in $q$ occur at lower $p^{\prime}$ values.

The stress paths for two CV tests are also included in Fig. 4. The onset of instability for the CV tests (indicated by an initial maximum in $q$ ) is marked by the star symbols, and the instability lines (IL), which connect the onset of instability and the origin, are superimposed for two cases with $e_{0}=0.6238$ and $e_{0}=0.6491$. Soon after, the onset of instability (start of decreases in $q$ ) becomes apparent (Fig. 4). Darve et al. (2007), Daouadji et al. (2010) and Chu et al. (2012) showed experimentally that a CSD test becomes unstable as soon as it crosses the instability line defined from a $\mathrm{CV}$ test having the same initial density.

A decrease in $q$ for CSD-500-0.6491-118 occurs at almost the same stress ratio as the onset of instability for CV-500-0.6491, agreeing with the observations from Darve et al. (2007), Daouadji et al. (2010) and Chu et al. (2012). The test CSD-500-0.6491-250 becomes unstable as soon as CSD conditions are imposed, as it had already crossed the IL before the isotropic unloading. The stress ratios at which the decrease in $q$ starts for CSD-500-0.6238-118 and CSD-500-0.6238-250 are below that for CV-500-0.6238 in the $q-p^{\prime}$ plane, suggesting that the test CV-500-0.6238 lost controllability before reaching an initial peak in $q$; moreover, fluctuations around the initial peak in $q$ are noted as well. In fact, the initial peak in $q$ does not indicate an effective failure, given that $q$ is seen to steadily increase again after reaching a minimum in $p^{\prime}$, becoming dilatant.

The axial strain rate plotted against time is presented in Fig. 5(a) for the CSD tests. For all tests, the strain rates are initially lower than $100 \mathrm{~s}^{-1}$ but sharply increase approaching the end of the tests. The densest sample experiences very low strain rates $\left(<1 \mathrm{~s}^{-1}\right)$ that yield a low $I\left(\leq 2.5 \times 10^{-3}\right)$ indicating quasi-static states (inertial forces are negligible). The increase in strain rate observed at the onset of instability, together with a decrease in $p^{\prime}$, leads to an increase in $I\left(>1 \times 10^{-3}\right)$, indicating that the system is moving from the quasi-steady regime into the dense flow regime (da Cruz et al., 2005). In the dense flow regime, the inertial forces become relevant and granular materials start flowing like a liquid (Jop et al., 2006). After collapse, the strain rate increases sharply for all samples tested with values ranging from $57.24 \mathrm{~s}^{-1}(I=0.062)$ to more than $390.6 \mathrm{~s}^{-1}$ $(I=0 \cdot 4241)$. An $I>0 \cdot 1$ indicates that the system is in the collisional dynamic regime where inertial forces are dominant.

Figure 5(b) shows the volumetric strain $\left(\varepsilon_{\mathrm{v}}\right)$ plotted against time for the CSD tests. For dense $\left(e_{0}=0.5533\right)$ and medium dense $\left(e_{0}=0.6238\right)$ samples, dilation takes place throughout the entire test. In contrast, the loose sample $\left(e_{0}=0 \cdot 6491\right)$ sheared from $q_{\mathrm{CSD}}=118 \mathrm{kPa}$ dilates for a short period of time after the CSD reaching a minimum value of $\varepsilon_{\mathrm{v}}$, after which the sample contracts. For the loose sample sheared from $q_{\mathrm{CSD}}=250 \mathrm{kPa}$, even though an isotropic unloading is taking place, the sample had already failed and thus immediately became contractant.

More details of the strain rate and $\varepsilon_{\mathrm{v}}$ at small strains are presented in Figs 5(c) and 5(d). The dense and medium dense sample contract initially, followed by an abrupt change to dilation as soon as the CSD conditions are imposed. Although its overall volumetric response is dilative, the dense sample initially extends in the axial strain direction until $\varepsilon_{1}$ reaches $0 \cdot 005 \%$, after which the dense sample becomes compressive again in the axial direction. The tendency for $\varepsilon_{1}$ to reverse diminished with a higher $q_{\mathrm{CSD}}$ and with an increase in $e_{0}$. Both the dense and medium dense samples with $q_{\mathrm{CSD}}=250 \mathrm{kPa}$ showed no reversal in $\varepsilon_{1}$, whereas the medium dense sample with $q_{\mathrm{CSD}}=118 \mathrm{kPa}$ showed a 

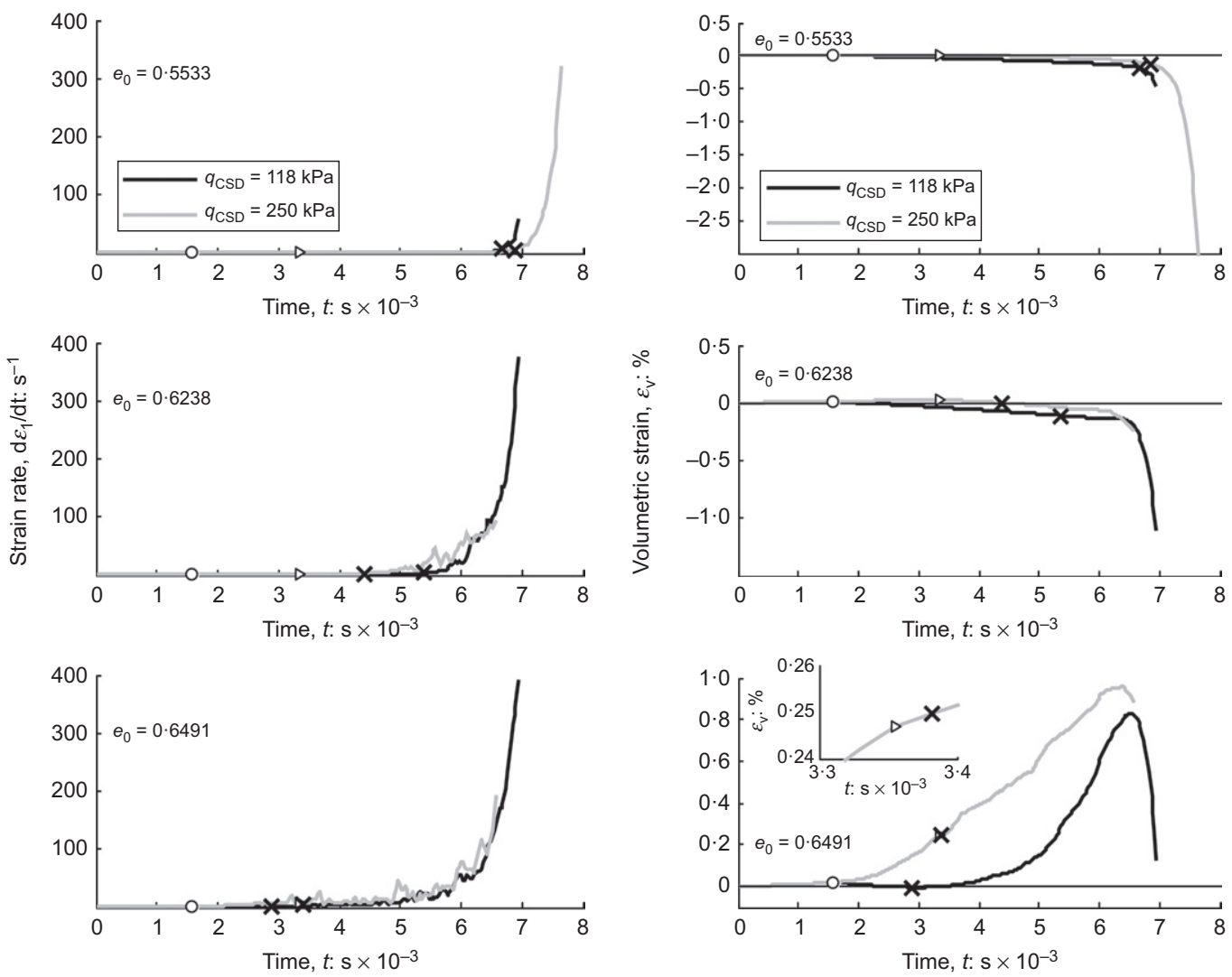

(a)

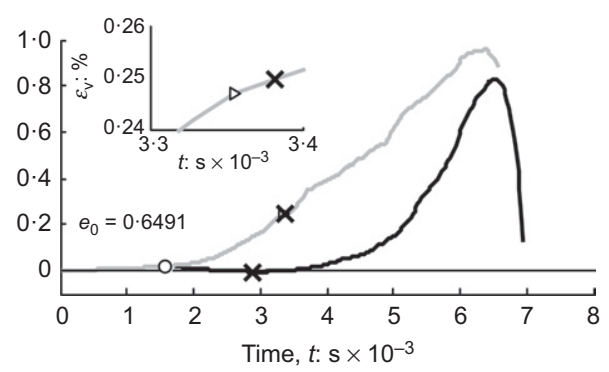

(b)
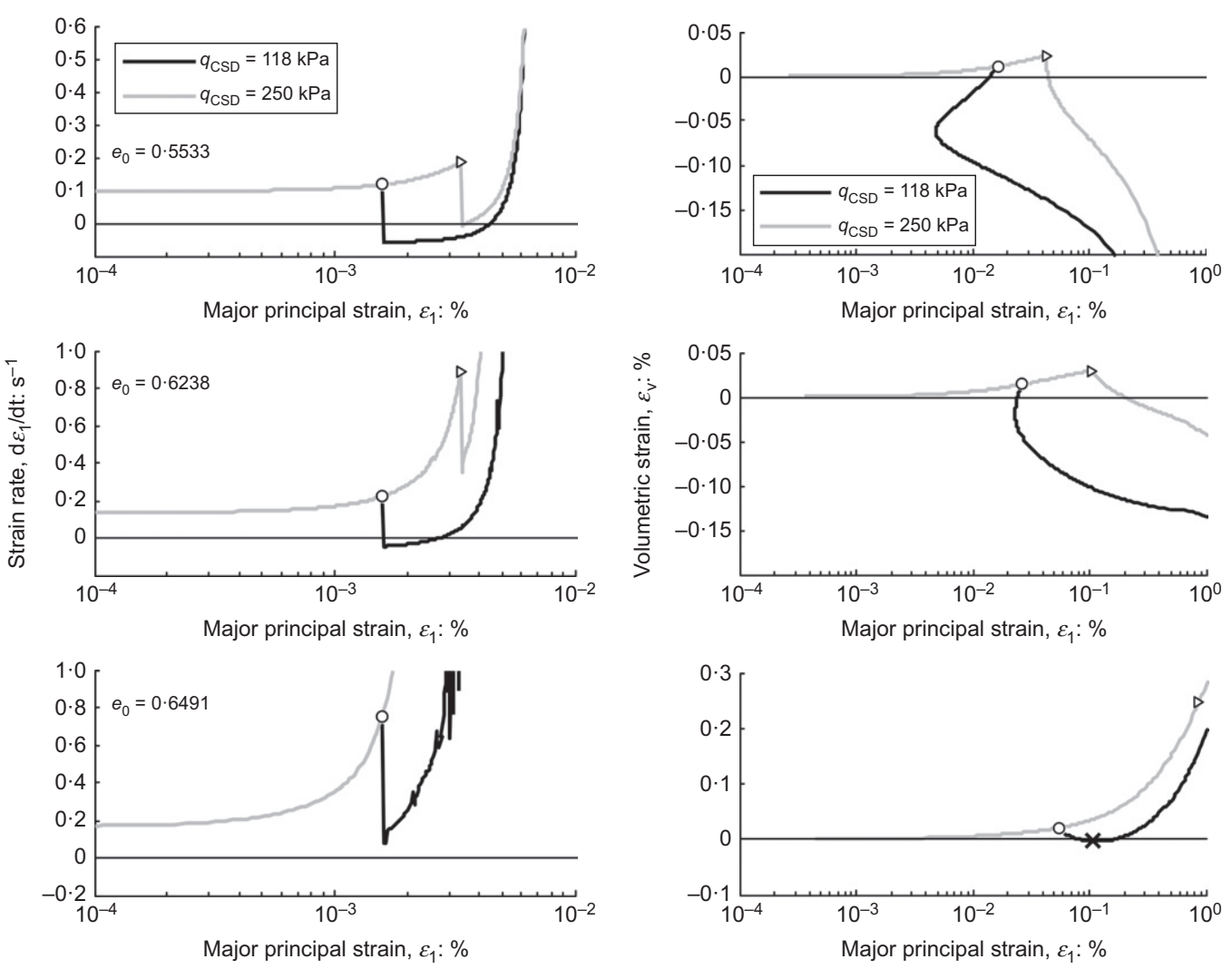

(c)

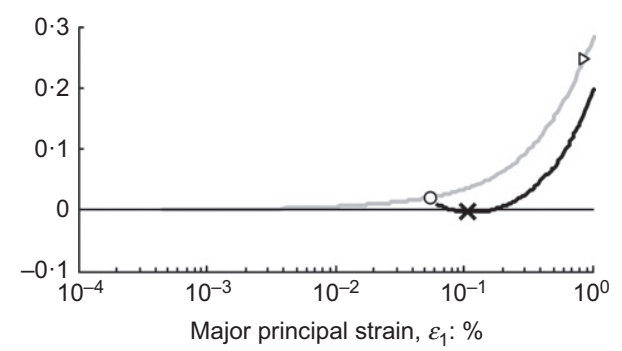

(d)

Fig. 5. (a) Strain rate plotted against time; (b) volumetric strain plotted against time; (c) strain rate plotted against axial strain for the CSD tests; and (d) volumetric strain plotted against axial strain. Cross markers indicate the onset of instability

negligible reversal in $\varepsilon_{1}$. Similar responses in terms of reversal of $\varepsilon_{1}$ were found by Darve et al. (2004) and Nicot et al. (2011). For the loose sample with $q_{\mathrm{CSD}}=118 \mathrm{kPa}$, a short period of dilation took place as soon as CSD conditions were imposed. The sample contracted again at around $0 \cdot 1 \%$ of $\varepsilon_{1}$ indicating instability, that is, an isotropic unloading is 

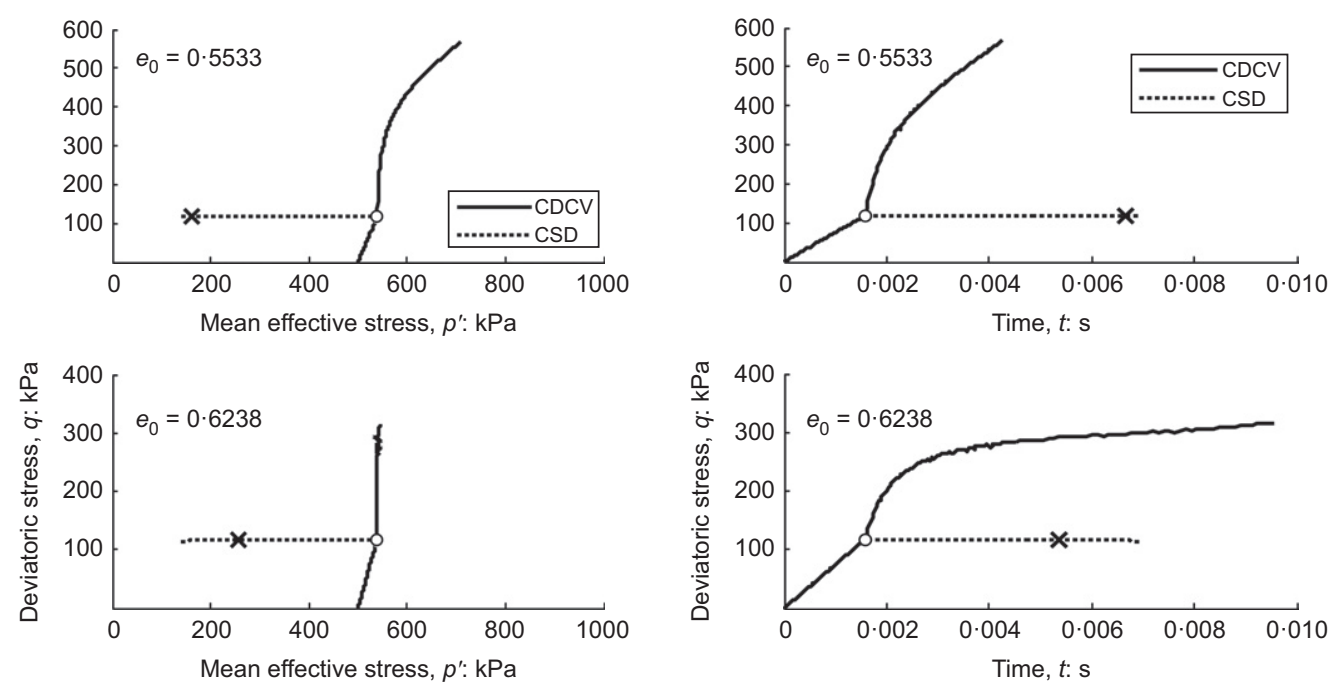

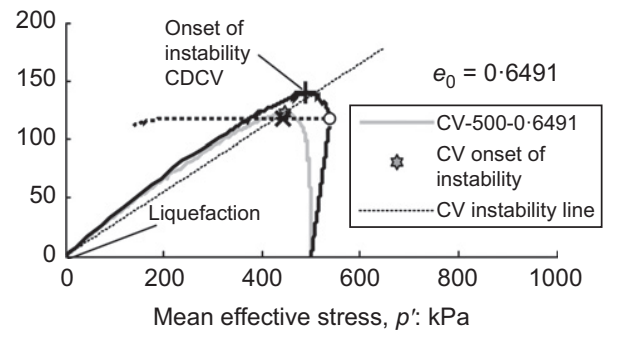

(a)

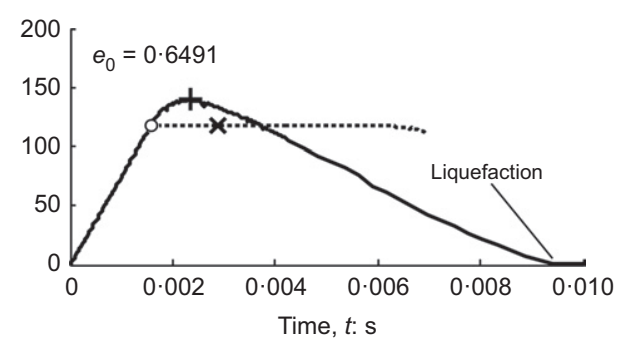

(b)

Fig. 6. Comparing the macro-scale responses between CSD test and CDCV tests: (a) $q$ plotted against $p^{\prime}$; (b) $q$ plotted against time. Cross markers indicate the onset of instability for CSD tests and plus sign markers indicate the onset of instability for the CDCV test

becoming contractant. At small strains, the loose sample with $q_{\mathrm{CSD}}=250 \mathrm{kPa}$ did not show either a reversal of $\varepsilon_{1}$ or a drop in $\varepsilon_{1}$. Although the inset figure at the bottom of Fig. 5(b) shows that the rate in contraction reduces after imposing the isotropic unloading for the loose sample with $q_{\mathrm{CSD}}=250$, no dilation was present in this sample given that it had already crossed the bifurcation domain boundary when CSD conditions took place.

Figure 5(a) shows how the sharp increase in strain rate coincides with the onset of instability. In Fig. 5(b), collapse, marked by the rapid development of volumetric strain, occurs immediately after the onset of instability for the dense sample. However, for the medium dense and loose samples, a longer period of shearing is required before collapse occurs. The volumetric behaviour after collapse is fairly similar in all samples considered, and is always related to a sudden and sharp drop of volumetric strain.

Figure 6(a) presents the macro response of the CDCV tests along with the CSD tests for direct comparison on the $q-p^{\prime}$ stress plane. During constant volume conditions, instability occurs once a local maximum in $q$ is reached. As indicated in the $q-p^{\prime}$ stress plane in Fig. 6(a), only the loose sample reached instability. Subsequently, it fully liquefied to $p^{\prime}=0 \mathrm{kPa}$ and $q=0 \mathrm{kPa}$. Both the medium dense and dense samples continued to show an increase in $q$ with a dilative tendency (increase in $p^{\prime}$ and $q$ ).

The bottom figure of Fig. 6(a) includes the stress path of a $\mathrm{CV}$ test sheared from the same initial void ratio, the instability line (joining the peaks in $q$ for the $\mathrm{CV}$ test with the origin) and the onsets of instability from the CV, CSD and CDCV tests. The onset of instability from the CDCV tests appears immediately after crossing the instability line and thus the time the CDCV test needed to become unstable is given by the time it took to reach the instability line. This result also suggests that, had the sample been switched to CV conditions after crossing the instability line, no time would have been allowed between imposing the $\mathrm{CV}$ conditions and failure. It is shown how the onsets of instability for three different tests are attained closely at the instability line, suggesting that, regardless of the kind of test (CV, CSD or CDCV), once the stress path crosses the instability boundary domain an onset of instability will be attained.

Figure 6(b) shows $q$ against time. As soon as the CDCV test is initiated, the three samples start to deviate from each other. The dense and medium dense samples show no sign of instability under CDCV conditions. A sharp increase in $q$ is observed for the dense sample, which diminishes as $e_{0}$ increases. The loose sample under CDCV conditions experienced the onset of instability earlier than the CSD test. By the time that the CSD attained its onset of instability, $q$ for the CDCV test was already lower than that for the CSD test. It was shown by Darve et al. (2007) how, once the stress path crossed the bifurcation domain boundary, failure would appear immediately. This bifurcation domain boundary corresponds to the instability line from a CV test.

Figure 7(a) shows the $e-p^{\prime}$ response for the CSD tests discussed above. The critical state line (indicated on the figure as CSL) obtained from those conventional drained and undrained triaxial tests described in Table 1 is overlaid and is represented by a linear relationship following Li \& Wang (1998). In all cases, the onset of instability occurs before reaching the critical state line. At the onset point of collapse, the failure mechanism is dilation regardless of the initial state. For the loose sample $\left(e_{0}=0 \cdot 6491\right)$, it initially contracts towards the critical state line after the onset of instability, and crosses the critical state line followed by dilation before collapsing. Similar observations in the $e-p^{\prime}$ plane from laboratory CSD tests have been reported by Chu \& Leong (2001). 

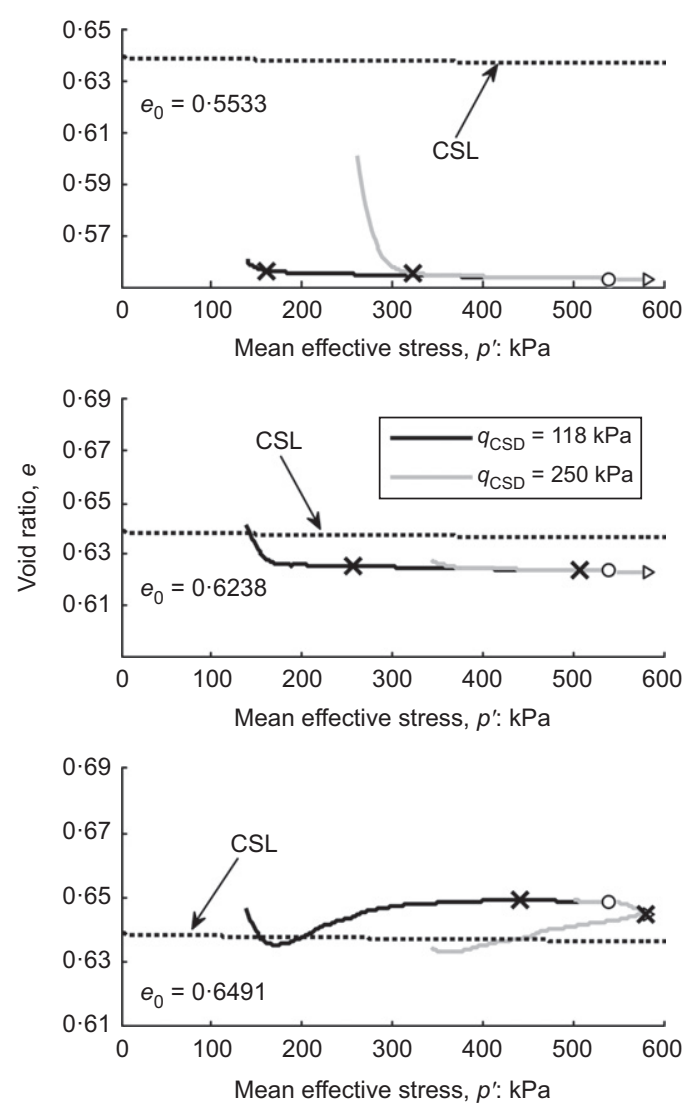

(a)

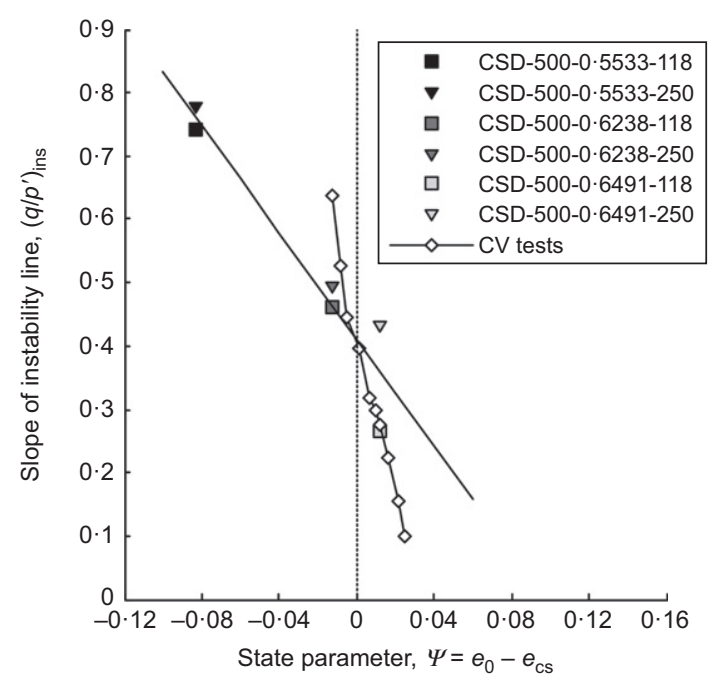

(b)

Fig. 7. (a) Void ratio $e$ plotted against mean effective stress $p^{\prime}$ during CSD shearing; (b) stress ratio at the onset of instability plotted against state parameter, showing CSD tests and CV tests. Cross markers in Fig. 7(a) indicate the onset of instability

Been \& Jefferies (1985) introduced the concept of the state parameter $(\psi)$, defined as the difference between the initial void ratio before shearing takes place $\left(e_{0}\right)$ and the void ratio at the critical state $\left(e_{\mathrm{cs}}\right)$ at the same $p^{\prime}$. A positive $\psi$ indicates a loose state with a tendency to contract, while a negative $\psi$ indicates a dense state with a tendency to dilate. Fig. 7(b) includes data points relating the stress ratio $\left(\eta=q / p^{\prime}\right)$ at the onset of instability and the state parameter for the CSD and $\mathrm{CV}$ tests (diamond symbols). In general, the data points from the CSD tests can be represented by a linear relationship. Data points corresponding to the onset of instability state for medium dense and loose samples in CSD tests are close to the data points for the $\mathrm{CV}$ tests.

Test CSD-500-0.6491-118 and test CV-500-0.6491 have the same $e_{0}$ and attain an almost identical relationship between stress ratio at the onset of instability and the initial state parameter. Data points corresponding to CSD tests deviate from the CV $\eta-\psi$ relationship. These tests find their onset of instability at higher $\eta$ (after crossing the CSL on the $q-p^{\prime}$ plane, as seen in Fig. 4). Although laboratory tests have shown that dense samples may also experience unstable behaviour under CSD conditions (Chu \& Leong, 2001; Ramos et al., 2012), it should be noted that for CV cases a $\eta-\psi$ relationship would only be valid up to a certain critical density (Lindenberg \& Koning, 1981), beyond which the soil would stop experiencing an onset of instability (in the present case $e_{0}=0.6238$ ). For this reason, data points showing the $\eta-\psi$ relationship at the onset of instability for the dense sample are not captured by the $\mathrm{CV}$ test $\eta-\psi$ relationship.

\section{Identifying the onset of instability}

A number of studies (Darve et al., 2004, 2007; Daouadji et al., 2010; Nicot et al., 2011; Hadda et al., 2013) have shown that the second-order work in terms of the conditions of stability (Hill, 1958) can serve as a good indicator of diffuse instability. Unlike localised instabilities (i.e. shear bands), diffuse instability results in displacement fields where strain patterns cannot be distinguished as in the case of instabilities present in CSD tests (Darve \& Roguiez, 1998; Nicot et al., 2011; Ramos et al., 2012).

Hill's condition of instability states that the stress-strain state is stable if the second-order work is strictly positive $\left(\mathrm{d}^{2} W=\mathrm{d} \sigma^{\prime} \mathrm{d} \varepsilon>0\right)$ for all changes in stress and strain (Darve et al., 2004). The second-order work for triaxial conditions is given by equation (1) (Sawicki \& Swidzinski, 2010), while Fig. 8 shows the second-order work defined here in equation (1) for the stress paths shown in Fig. 3.

$$
\mathrm{d}^{2} W=\mathrm{d} \varepsilon_{\mathrm{v}} \mathrm{d} p^{\prime}+\mathrm{d} \varepsilon_{\mathrm{q}} \mathrm{d} q
$$

Imposing a CSD stress path implies $\mathrm{d} q=0$, and $\mathrm{d} p^{\prime}<0$, thus an unstable state will be associated with $\varepsilon_{\mathrm{v}}$ passing through an extremum (Darve et al., 2004). Thus, for CV paths, an unstable state will be associated with $q$ passing through an extremum.

In this study, in the case of CSD conditions only isotropic loading is imposed, whereas CV tests are loaded by strain control. These test conditions follow those that are usually used in laboratory experiments (Chu et al., 2003, 2012). Experiments conducted by $\mathrm{Chu}$ et al. (2012) and Chu et al. (2003) showed $\mathrm{d} \varepsilon_{\mathrm{v}}>0$ for loose samples and $\mathrm{d} \varepsilon_{\mathrm{V}}<0$ for dense samples both before and after the onset of instability. Both cases were also obtained in this study (Fig. 5), implying that $\mathrm{d}^{2} W$ can remain positive during CSD tests even after the system becomes unstable. $\mathrm{d}^{2} W$ is plotted against time for the dense sample with $q_{\mathrm{CSD}}=118 \mathrm{kPa}$ in Fig. 8 where the observations of the second-order work do not coincide with Hill's stability condition; that is, the system is clearly both unstable and has $\mathrm{d}^{2} W>0$. On the other hand, the loose sample with $q_{\mathrm{CSD}}=118 \mathrm{kPa}$, which experienced an extremum in $\varepsilon_{\mathrm{v}}$ has its onset of instability at the minimum value of $\varepsilon_{\mathrm{v}}$, leading to the occurrence of negative values of $\mathrm{d}^{2} W$ (as indicated by cross markers in Fig. 8(a)) that matches with the start of decrease in $q$.

Darve et al. (2004) showed theoretically, and Daouadji et al. (2010) showed experimentally, that CSD tests and CV tests have the same bifurcation criteria. The peak in $q$ for test CDCV-500-0.6491-118 and the onset of instability for the test CSD-500-0.6491-118 that corresponds to its extrema of 


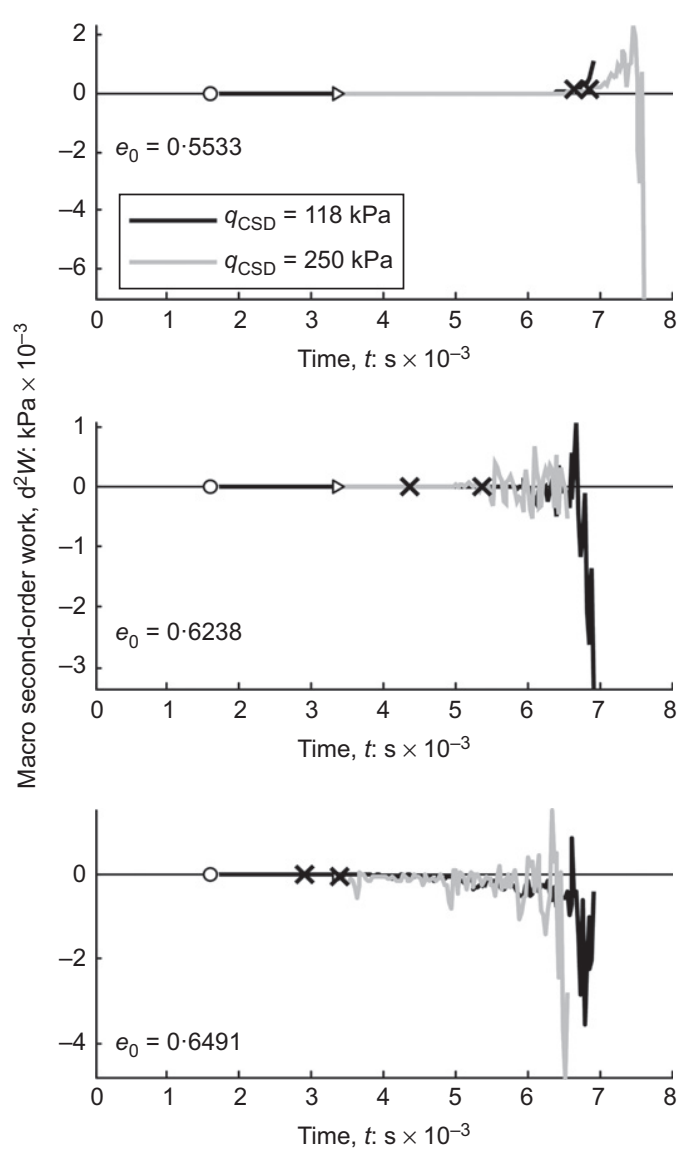

(a)
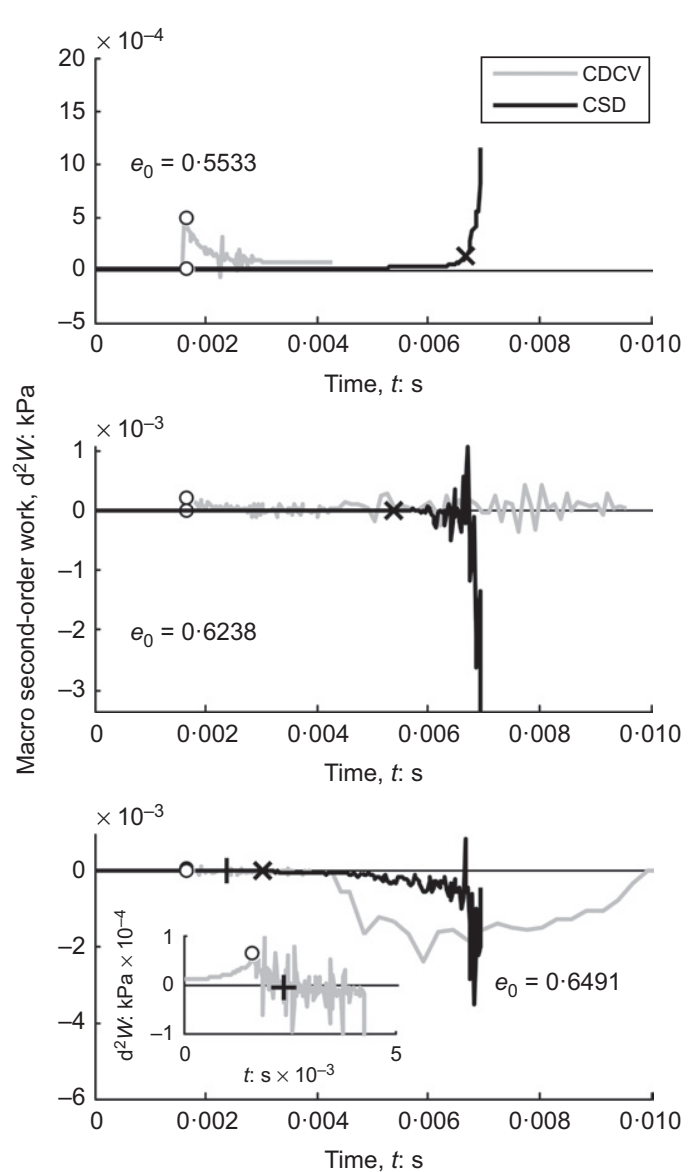

(b)

Fig. 8. Macro-scale second-order work plotted against time for (a) all CSD tests and (b) CSD tests with $q_{\mathrm{CSD}}=118 \mathrm{kPa}$ and $\mathrm{CDCV}$ tests. Cross markers indicate the onset of instability for CSD tests and plus sign markers indicate the onset of instability for the CDCV test

$\varepsilon_{\mathrm{v}}$, which are plotted in the $q-p^{\prime}$ plane in Figs 4 and 6(a), are coincident with the instability line from the CV test. As these samples have the same initial density, the DEM data confirm these earlier observations; this also indicates that stress paths such as CDCV have the same bifurcation criteria as CV tests, providing additional support for the observation of Darve et al. (2004).

As $e_{0}$ increases, the fluctuations in $\mathrm{d}^{2} W$ become more evident (refer to the loose sample with $q_{\mathrm{CSD}}=250 \mathrm{kPa}$ ) and the onsets of instability are seen to appear sooner. Referring to Fig. 8(b) the dense sample sheared under CDCV conditions shows positive values of $\mathrm{d}^{2} W$ throughout the test. Fluctuations between positive and negative values of $\mathrm{d}^{2} W$ are present for the CDCV test sheared from $e_{0}=0.6238$; these are mostly related to small fluctuations in $q . \mathrm{d}^{2} W$ shows negative values for the loose CDCV test around the onset of instability (maximum in $q$ ) followed by a drop in $\mathrm{d}^{2} W$ after $4 \mathrm{~ms}$, which is associated with a decay in $q$.

Hadda et al. (2013) proposed the microscopic formulation for the second-order work defined by equation (2).

$$
W_{2}^{\mathrm{p}}=\sum_{c \in V} \mathrm{~d} f_{i}^{\mathrm{c}} \mathrm{d} \boldsymbol{l}_{i}^{\mathrm{c}}+\sum_{p \in V} \mathrm{~d} f_{i}^{\mathrm{p}} \mathrm{d} x_{i}^{\mathrm{p}}
$$

where $\boldsymbol{l}^{\mathrm{c}}$ is the branch vector connecting the centres of contacting particles within the volume $V, f^{\mathrm{c}}$ is the inter-particle contact force, $x^{\mathrm{p}}$ is the coordinates of particle $p$, and $f^{\mathrm{p}}$ is the resultant force for particle $p$. The first term in equation (2) is the contact-based second-order work, while the second term is the particle-based second-order work.

The second term of equation (2) is negligible if the system is in equilibrium (Nicot et al., 2011; Hadda et al., 2013).
However, once particles within the sample move rapidly, the second term of equation (2) becomes important. An inspection of the second-order work at a particle scale throughout the CSD tests can serve as an appropriate indicator of the onset of instability. Darve et al. (2004) found this approach useful when determining the onset of instabilities in DEM simulations of slopes, where the appearance of negative values of the second-order work coincided with instabilities within the slope. Here the appearance of negative values of $W_{2}^{\mathrm{p}}$ within the sample corresponds to the onset of instability.

Figure 9 presents the second-order work at a particle scale $\left(W_{2}^{\mathrm{p}}\right)$ against time for the six CSD tests conducted. Referring to Fig. 5(b), there is no clear maximum in the volumetric strains for the CSD tests with $e_{0}$ of 0.5533 or 0.6238 and so for these CSD tests, the onset of instability was identified from the inability of the system to sustain the imposed $q$ by considering $\mathrm{d} q / \mathrm{d} p^{\prime}$ calculated between neighbouring data output points as illustrated in Fig. 9. The ratio $\mathrm{d} q / \mathrm{d} p^{\prime}$ was zero or very small $\left(\sim 10^{-7}\right)$ before the onset of instability; following a parametric study, a threshold value of $\mathrm{d} q / \mathrm{d} p^{\prime}$ of $1 \times 10^{-3}$ (i.e. $\mathrm{d} q / \mathrm{d} p^{\prime}>1 \times 10^{-3}$ ) was taken to identify a reduction in $q$, marking the onset of instability. These points are marked on Fig. 4. It is clear that the instability points for tests CSD-500-0.6238-118 and CSD-500-0.6238-250 do not lie on the instability line for the CV test with $e_{0}$ of 0.6238 , that is, using these criteria to identify instability. These data do not conform to the theory proposed by Darve et al. (2004). The lack of agreement may be attributable to the use of $\sigma_{1}^{\prime}$ as a stress variation controlled loading rather than a volume variation controlled loading. However, the points identified using $\mathrm{d} q / \mathrm{d} p^{\prime}>1 \times 10^{-3}$ are consistent with the appearance of 

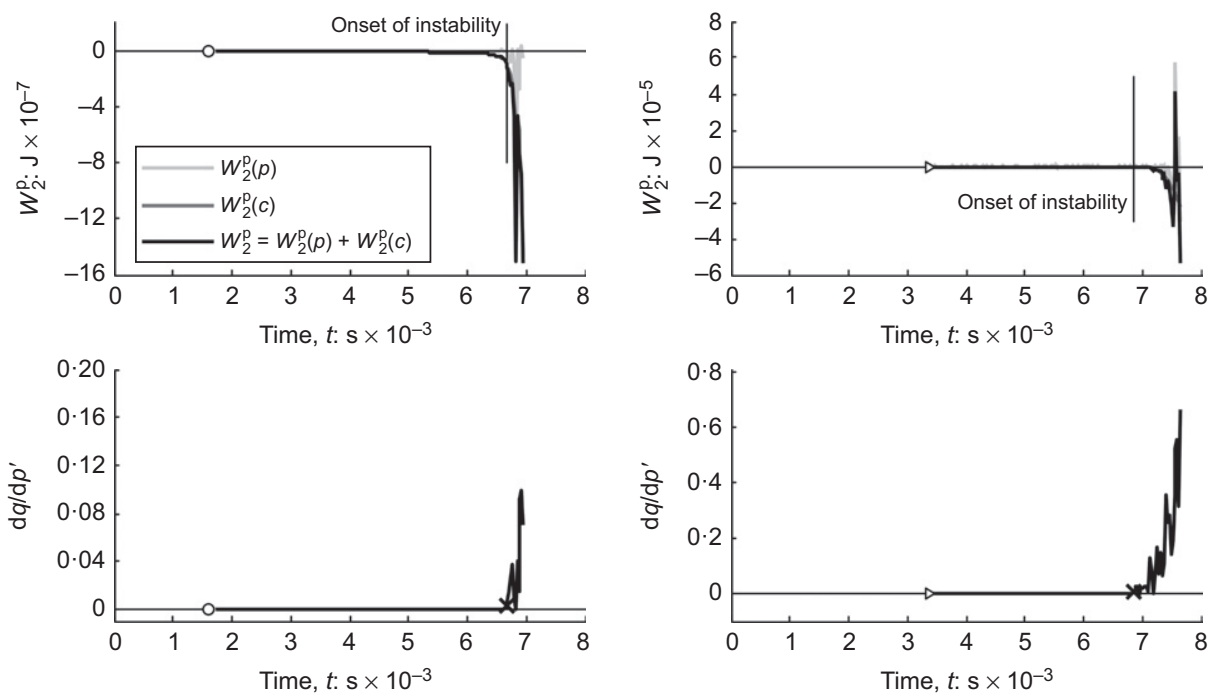

(a)

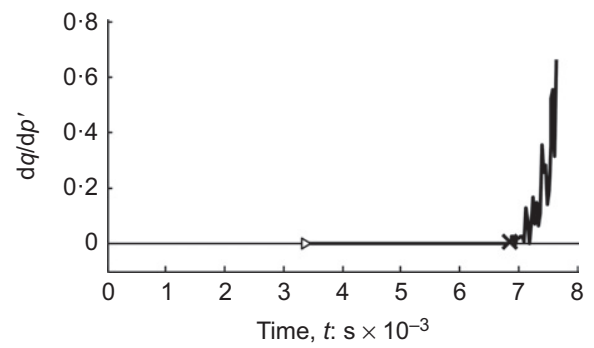

(b)
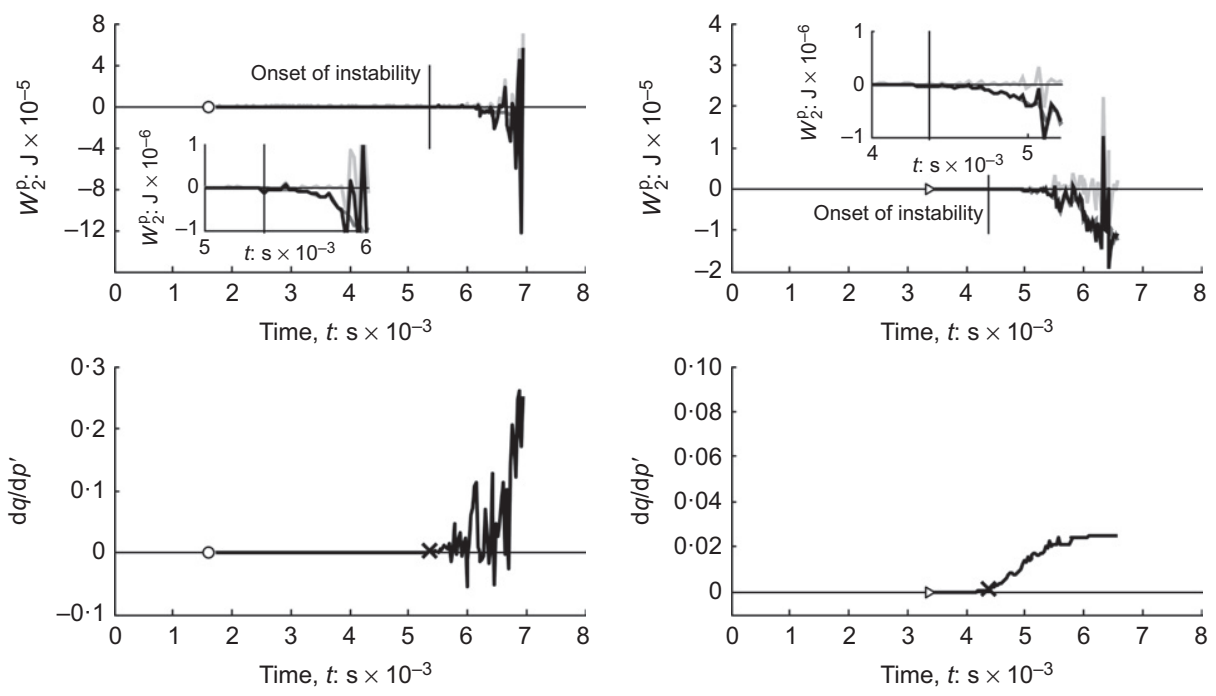

(c)

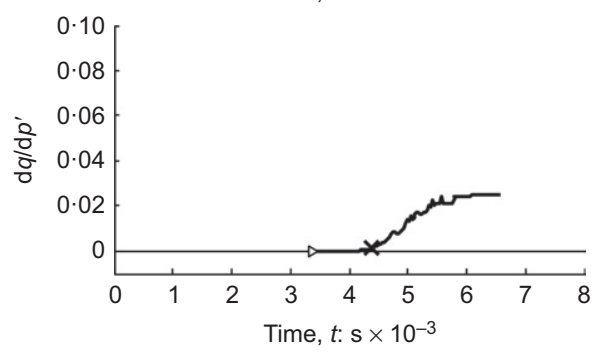

(d)
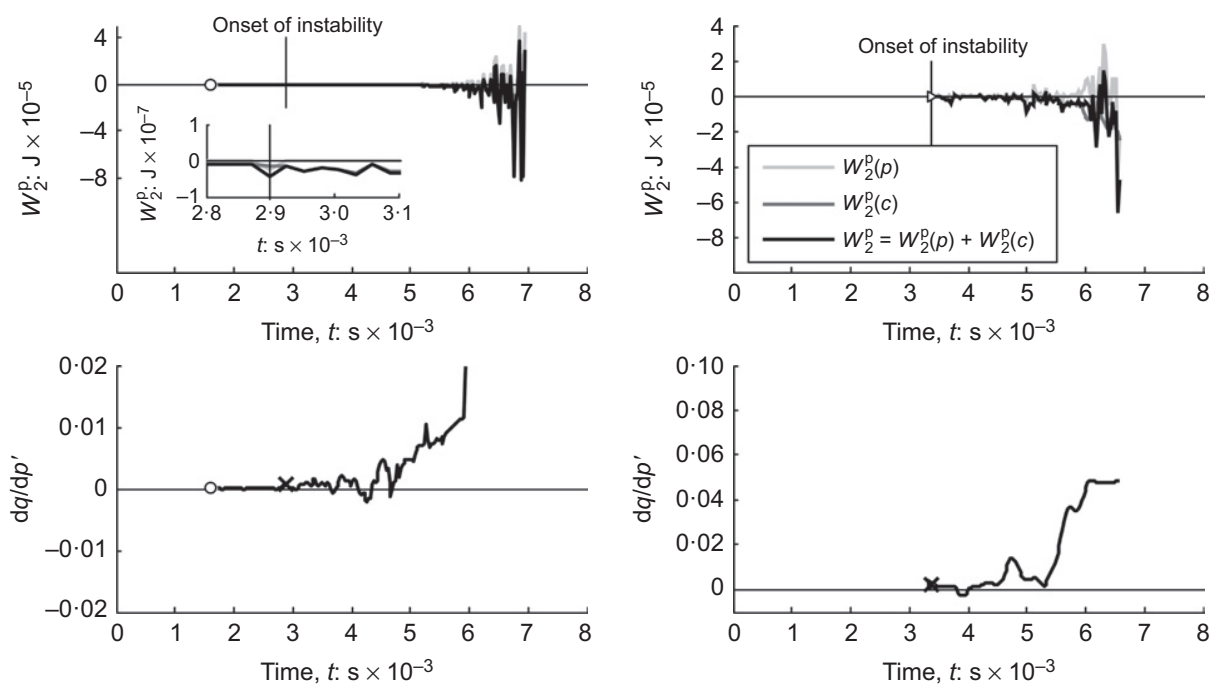

(e)

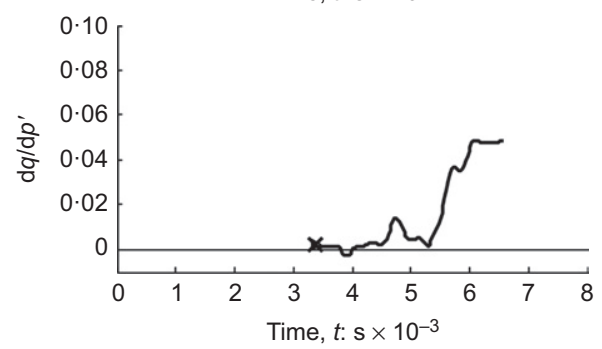

(f)

Fig. 9. Micro-scale second-order work and gradient $d q / d p^{\prime}$ plotted against time for the CSD tests: (a) CSD-500-0.5533-118; (b) CSD-500-0.5533-250; (c) CSD-500-0.6238-118; (d) CSD-500-0.6238-250; (e) CSD-500-0.6491-118; (f) CSD-500-0.6491-250. Cross markers and vertical lines indicate the onset of instability

negative values and initiation of fluctuations in $W_{2}^{p}$ Although for the loose sample with $q_{\mathrm{CSD}}=118 \mathrm{kPa}$ an extremum in $\varepsilon_{\mathrm{v}}$ allowed the recognition of the onset of instability, $W_{2}^{p}$ and $\mathrm{d} q / \mathrm{d} p^{\prime}$ are also plotted for this case, to illustrate that its onset of instability also coincided with negative values in $W_{2}^{\mathrm{p}}$ and rise of $\mathrm{d} q / \mathrm{d} p^{\prime}$. As it becomes 
more difficult for samples to sustain the imposed $q, \mathrm{~d} q / \mathrm{d} p^{\prime}$ starts to rise and at the same time $W_{2}^{p}$ starts to exhibit small-amplitude oscillations around zero. However, once $W_{2}^{p}$ becomes negative, $\mathrm{d} q / \mathrm{d} p^{\prime}$ shows abrupt and noticeable changes. While the contact-based second-order work $\left(W_{2}^{\mathrm{p}}(c)\right)$ decreases consistently to negative values after the initiation of instability, the particle-based component of second-order work $\left(W_{2}^{\mathrm{p}}(p)\right)$ oscillates between positive and negative values, and this oscillation accounts for the oscillation of the overall second-order work $W_{2}^{p}$. This agrees with Darve et al. (2004), who noted that particles with positive $W_{2}^{\mathrm{p}}$ remain within a system even after instability has taken place.

The response for the loosest sample sheared under CDCV and CSD conditions is included in Fig. 10, which considers variations in $W_{2}^{\mathrm{p}}$ and $\mathrm{d} q / \mathrm{d} p^{\prime}$ with time. The response of $W_{2}^{\mathrm{p}}$ for the CDCV test shows no fluctuations after the onset of instability, with almost negligible negative values, in contrast to the CSD test. The very small negative values of $W_{2}^{\mathrm{p}}$ are associated with fluctuations of $\mathrm{d} q / \mathrm{d} p^{\prime}$ between negative and positive values. Conversely, fluctuations of $W_{2}^{\mathrm{p}}$ between negative and positive values for CSD tests are linked to an increase in $\mathrm{d} q / \mathrm{d} p^{\prime}$. From Figs 8 and 10 it is evident that the calculated macroscopic and particle-based second-order work are closely related, having changes in sign or fluctuations taking place virtually at the same instant, in agreement with Darve et al. (2007).

Figures 11(a) and 11(b) show how the proportions of particles and contacts carrying negative $W_{2}^{\mathrm{p}}$ change with time for all six CSD tests. For all cases, at the onset of CSD conditions, the proportion of particles with negative $W_{2}^{p}$ does not exceed $48 \%$ and this proportion remains almost constant from the onset of instability until the end of test. However, the proportion of contacts carrying negative $W_{2}^{\mathrm{p}}$ is dependent on packing density and stress level. When the CSD conditions are imposed and both packing density and stress level increase, a higher proportion of contacts with negative $W_{2}^{\mathrm{p}}$ are observed. At the onset of instability, the proportion
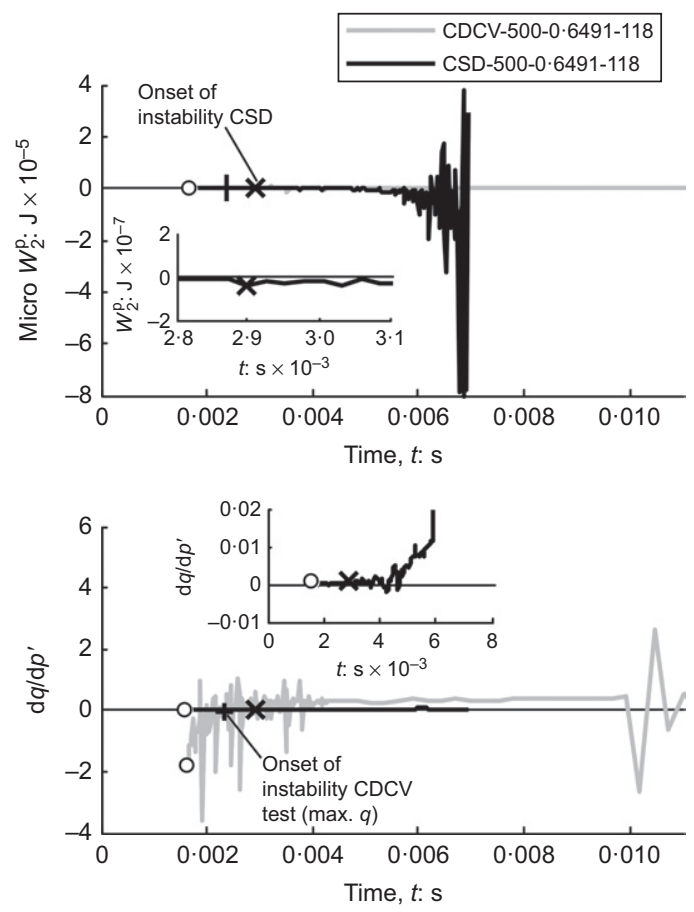

Fig. 10. Comparison of micro-scale second-order work and gradient $\mathrm{d} q / \mathrm{d} p^{\prime}$ plotted against time between CDCV and CSD tests. Cross markers indicate the onset of instability for CSD tests and plus sign markers indicate the onset of instability for the CDCV test of contacts with negative $W_{2}^{p}$ for the dense samples exceeds $60 \%$. For the medium dense and loose samples the proportion of contacts with negative $W_{2}^{p}$ is approximately $60 \%$ at the onset of instability, with the exception of the loose sample with $q_{\mathrm{CSD}}=250 \mathrm{kPa}$, where this proportion reaches $60 \%$ at $2 \mathrm{~ms}$, which is about the time the specimen crossed its bifurcation domain boundary. Close to the onset of instability there is a change in the slope in the plots illustrated in Fig. 11, with the post-instability slopes reducing as packing density increases.

\section{Micro-mechanical response}

In an attempt to understand the physical basis of instability during CSD tests, two particle-scale parameters were analysed: the structural anisotropy (geometrical and mechanical anisotropies) and the coordination number. Satake (1982) defined the fabric tensor as follows

$$
\boldsymbol{\Phi}_{i j}=\frac{1}{N_{\mathrm{c}}} \sum_{1}^{N_{\mathrm{c}}} n_{i} n_{j}
$$

where $N_{\mathrm{c}}$ is the total number of contacts and $n_{i}$ is the unit contact normal. The largest, intermediate and smallest eigenvalues of the fabric tensor are denoted as $\Phi_{1}, \Phi_{2}$ and $\Phi_{3}$, respectively. The deviatoric fabric, $\Phi_{1}-\Phi_{3}$, describes the degree of structural anisotropy.

Rothenburg \& Bathurst (1989) analytically proved that the stress ratio is related to different sources of anisotropy including geometrical anisotropy $\left(a_{\mathrm{c}}=15 / 2\left(\Phi_{1}-\Phi_{3}\right)\right)$, normal contact force anisotropy $\left(a_{\mathrm{n}}\right)$ and tangential contact force anisotropy $\left(a_{\mathrm{t}}\right)$, of which $a_{\mathrm{c}}$ and $a_{\mathrm{n}}$ are dominant. The definition of $a_{\mathrm{n}}$ used here follows Rothenburg \& Bathurst (1989) and Guo \& Zhao (2013), with the average normal contact force tensor being expressed by equation (4) (where $\boldsymbol{\Phi}_{i j}^{\prime}$ is the deviatoric part of $\left.\boldsymbol{\Phi}_{i j}\right)$ and its probability distribution given by equation (5). $a_{\mathrm{n}}$ is related to the second invariant of $a_{i j}^{\mathrm{n}}=(15 / 2) \boldsymbol{F}_{i j}^{\prime \mathrm{n}} / \overline{f^{0}}$ as $a_{\mathrm{n}}=\sqrt{(3 / 2) a_{i j}^{\mathrm{n}} a_{i j}^{\mathrm{n}}}$, where $\overline{f^{0}}=\boldsymbol{F}_{i i}^{\mathrm{n}}$ is the average normal contact force calculated considering the entire $V$, different from the mean normal contact force averaged over all contacts.

$$
\begin{aligned}
\boldsymbol{F}_{i j}^{\mathrm{n}} & =\frac{1}{4 \pi} \int_{V} \overline{f_{\mathrm{n}}}(V) n_{i} n_{j} \mathrm{~d} V \\
& =\frac{1}{N_{\mathrm{c}}} \sum_{1}^{N_{\mathrm{c}}} \frac{f_{\mathrm{n}} n_{i} n_{j}}{1+(15 / 2) \boldsymbol{\Phi}_{i j}^{\prime} n_{k} n_{l}}
\end{aligned}
$$

$$
\overline{f_{\mathrm{n}}}(V)=\overline{f^{0}}\left[1+a_{i j}^{\mathrm{n}}\right]
$$

Figures 12(a) and 12(b) plot $a_{\mathrm{c}}$ and $a_{\mathrm{n}}$ against time, respectively, for the CSD tests shown in Fig. 4. The initial $a_{\mathrm{c}}$ is close to zero as all the samples were initiated from an isotropic state. Before the CSD takes place (i.e. during constant $\sigma_{3}^{\prime}$ shearing), $a_{\mathrm{c}}$ is seen to slowly increase. Once the CSD conditions are imposed, $a_{\mathrm{c}}$ increases sharply. A change in the variation of $a_{\mathrm{c}}$ with time seems to take place around the onset of instability. At the onset of instability, dense samples have attained higher values of $a_{\mathrm{c}}$ than loose samples and after onset of instability an abrupt increase in $a_{\mathrm{c}}$ occurs, which is maintained until collapse. Although $q$ remains virtually constant, $a_{\mathrm{c}}$ does vary during shearing. Even after the onset of instability, $a_{\mathrm{c}}$ continues to increase while $q$ begins to decrease; that is, contacts continue rearranging towards the loading direction in order to sustain the imposed $q$ value.

Before the initiation of the CSD tests, $a_{\mathrm{n}}$ increases linearly. The rate of increase of $a_{\mathrm{n}}$ is clearly affected by the imposition 


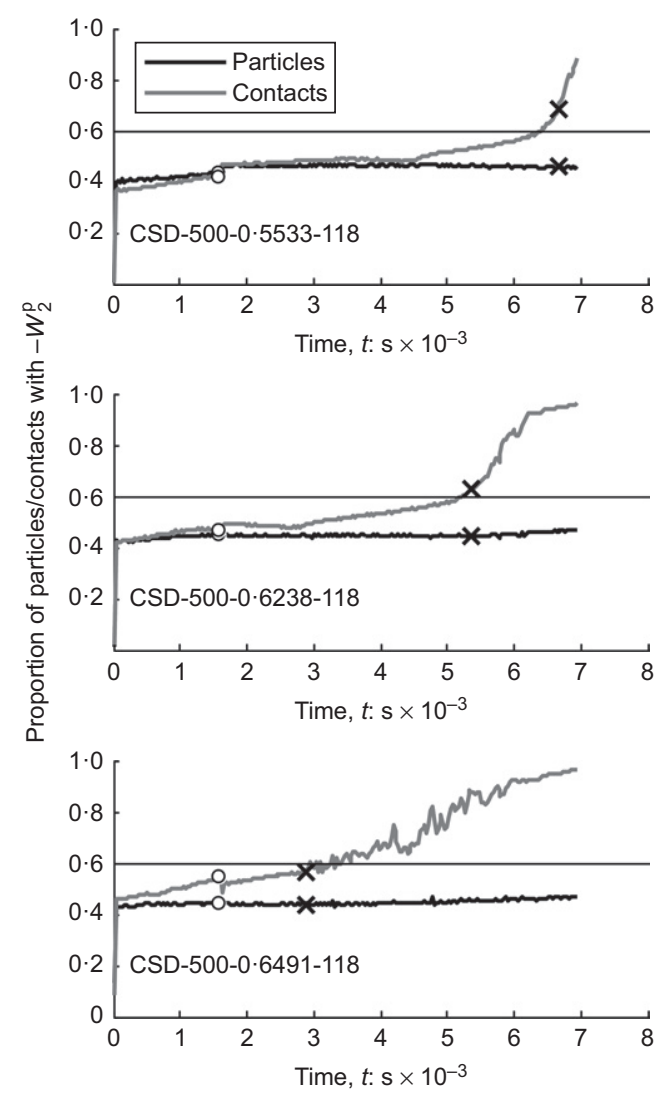

(a)

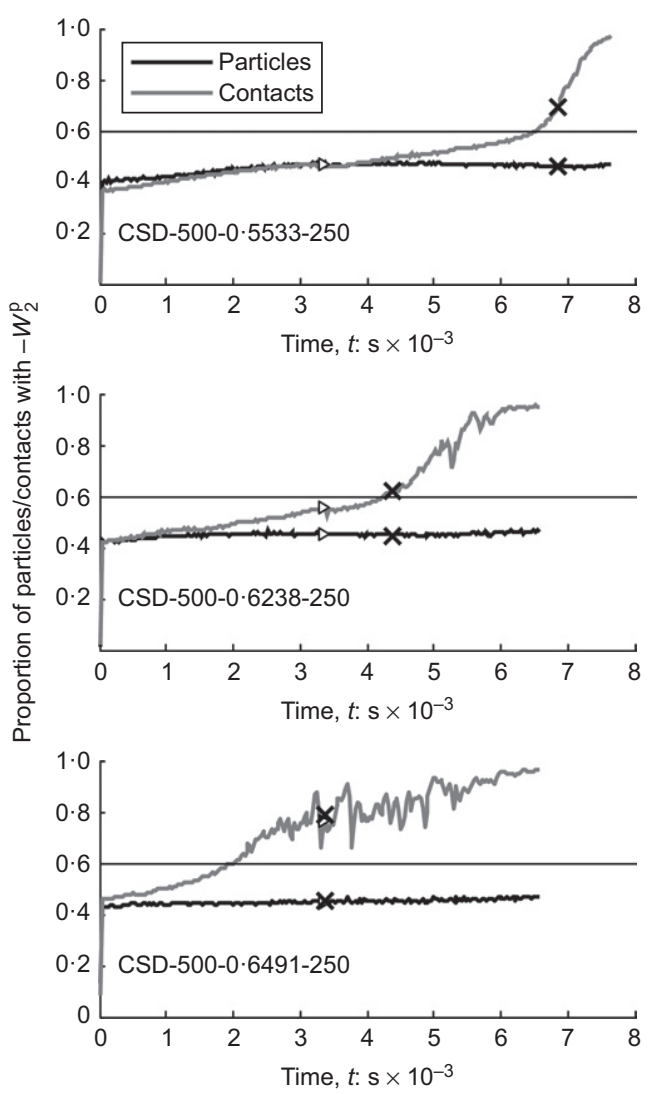

(b)

Fig. 11. Proportion of particles and contacts carrying negative micro second-order work: (a) $q_{\mathrm{CSD}}=118 \mathrm{kPa}$ and (b) $q_{\mathrm{CSD}}=250 \mathrm{kPa}$. Circle and triangle markers indicate the onset of CSD conditions for $q_{\mathrm{CSD}}=118 \mathrm{kPa}$ and $q_{\mathrm{CSD}}=250 \mathrm{kPa}$, respectively. Cross markers indicate the onset of instability

of the CSD stress path. The manner in which $a_{\mathrm{n}}$ increases depends on $e_{0}$ and $q_{\mathrm{CSD}}$ : dense and medium dense samples with $q_{\mathrm{CSD}}=118 \mathrm{kPa}$ show an exponential increase, whereas dense and medium dense samples with $q_{\mathrm{CSD}}=250 \mathrm{kPa}$ and both loose samples present a linear increase prior to the onset of instability, the rate of which is affected by $e_{0}$. An abrupt drop in $a_{\mathrm{n}}$ is seen closer to the onset of instability as the initial packing density increases, with dense samples showing a larger drop than loose samples. The onset of instability corresponds to a sudden reduction in normal contact force anisotropy, demonstrating that the system is less capable of sustaining the imposed deviatoric load while the system is at the same time losing $p^{\prime}$. In conjunction with Figs 13(a) and 13(b), it could be inferred that the overall increase of $q / p^{\prime}$ post the onset of instability is a consequence of the interplay between $a_{\mathrm{c}}$ and $a_{\mathrm{n}}$.

The coordination number, defined as $Z=2 N_{\mathrm{c}} / N_{\mathrm{p}}$ where $N_{\mathrm{p}}$ is the number of particles, is shown in Figs 12(c) and 12(d) for the cases of $q_{\mathrm{CSD}}=118 \mathrm{kPa}$ and $q_{\mathrm{CSD}}=250 \mathrm{kPa}$, respectively. Slightly modified definitions of the coordination number are also considered: the number of contacts that are contributing to $W_{2}^{\mathrm{p}}\left(Z_{W_{2}^{\mathrm{p}}}\right)$ (contacts that remained present after two consecutive data outputs), the number of contacts having positive $W_{2}^{\mathrm{p}}\left(Z_{+} W_{2}^{\mathrm{p}}\right)$ and the number of contacts having negative $W_{2}^{\mathrm{p}}\left(Z_{-W_{2}^{\mathrm{p}}}\right)$. In each case the number of contacts is normalised by the total number of particles in the system. As shearing takes place there is a quick drop in $Z_{+W_{2}^{\text {p }}}$ together with a sudden increase of $Z_{-} W^{\text {p }}$ that results in an almost constant $Z$. After the CSD conditions have been imposed, $Z_{W^{\mathrm{p}}}$ decreases notably at a rate that depends on $e_{0}$. At the onset of instability for all cases $Z_{W_{2}^{\mathrm{p}}}$ and $Z_{-W_{2}^{\mathrm{p}}}$ appear to be approaching a value of $Z=4$ leaving $Z_{+W_{2}^{\mathrm{p}}}$ below $Z=1$. When collapse starts, a sharper drop in $Z_{W_{2}^{\mathrm{p}}}^{2}$ is observed with values either close to $Z=4$ or below. $Z_{+} W^{2}$ is seen close to zero at collapse, while $Z_{-W^{\text {p }}}$ appears clóser to $Z=4$ but still below 4 . Fluctuations in $Z$ are also present which start appearing as the packing density and stress level increase. Ning et al. (2013) demonstrated an absence of strain localisation in their CSD tests by showing the distribution of the coordination number $(Z)$ along a vertical plane. Contour plots of $Z$ on a vertical plane at the onset of instability and at end of test (collapse) for a dense sample (CSD-500-0.5533-250) following a CSD path are given in Fig. 13. It is clear that at both stages the specimen shows an effectively homogeneous distribution of the coordination number, indicating that the mode of instability is diffuse.

To illustrate the influence of contact and normal contact force anisotropy for contacts carrying either negative or positive $W_{2}^{\mathrm{p}}$. Fig. 14 presents contact rose diagrams for a dense and a loose sample at the onset of instability with a stress level from $q_{\mathrm{CSD}}=250 \mathrm{kPa}$. The radial length of each bin gives the number of contacts present within the angle defining the bin. The shading of each bin is proportional to the average normal contact force that is oriented in that bin. Regardless of contacts carrying either negative or positive $W_{2}^{\mathrm{p}}$, a larger number of contacts being aligned in the vertical direction (major principal stress direction) are observed from the dense and loose samples. Fig. 14 also shows that contacts carrying either negative or positive $W_{2}^{\mathrm{p}}$ present a similar average normal contact force for each direction. However, owing to the higher number of contacts with negative $W_{2}^{p}$, at the onset of instability, contacts with negative $W_{2}^{\mathrm{p}}$ are carrying a larger amount of normal force mostly aligned in the vertical 

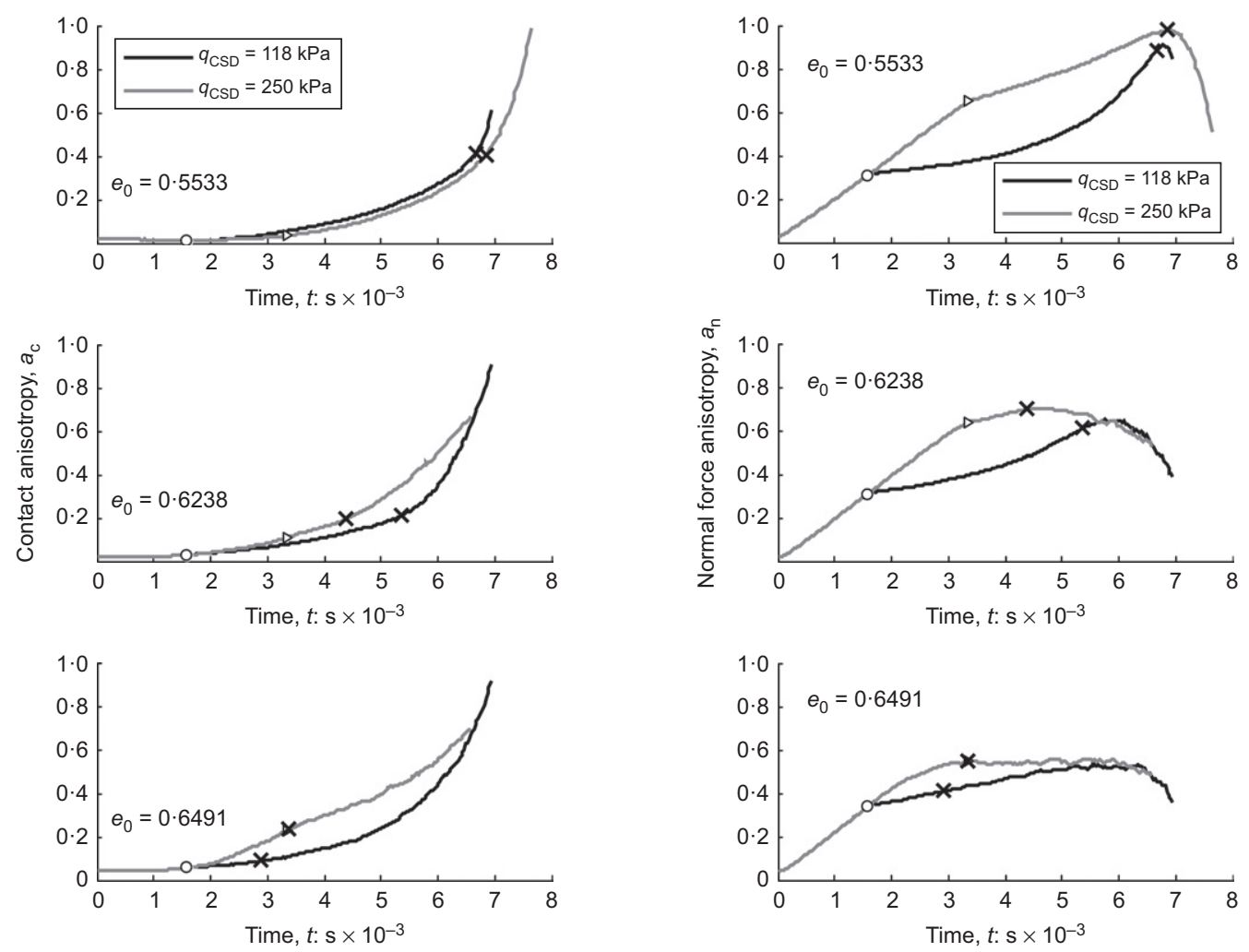

(a)

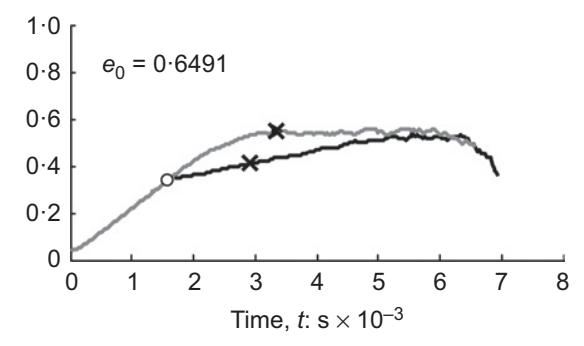

(b)
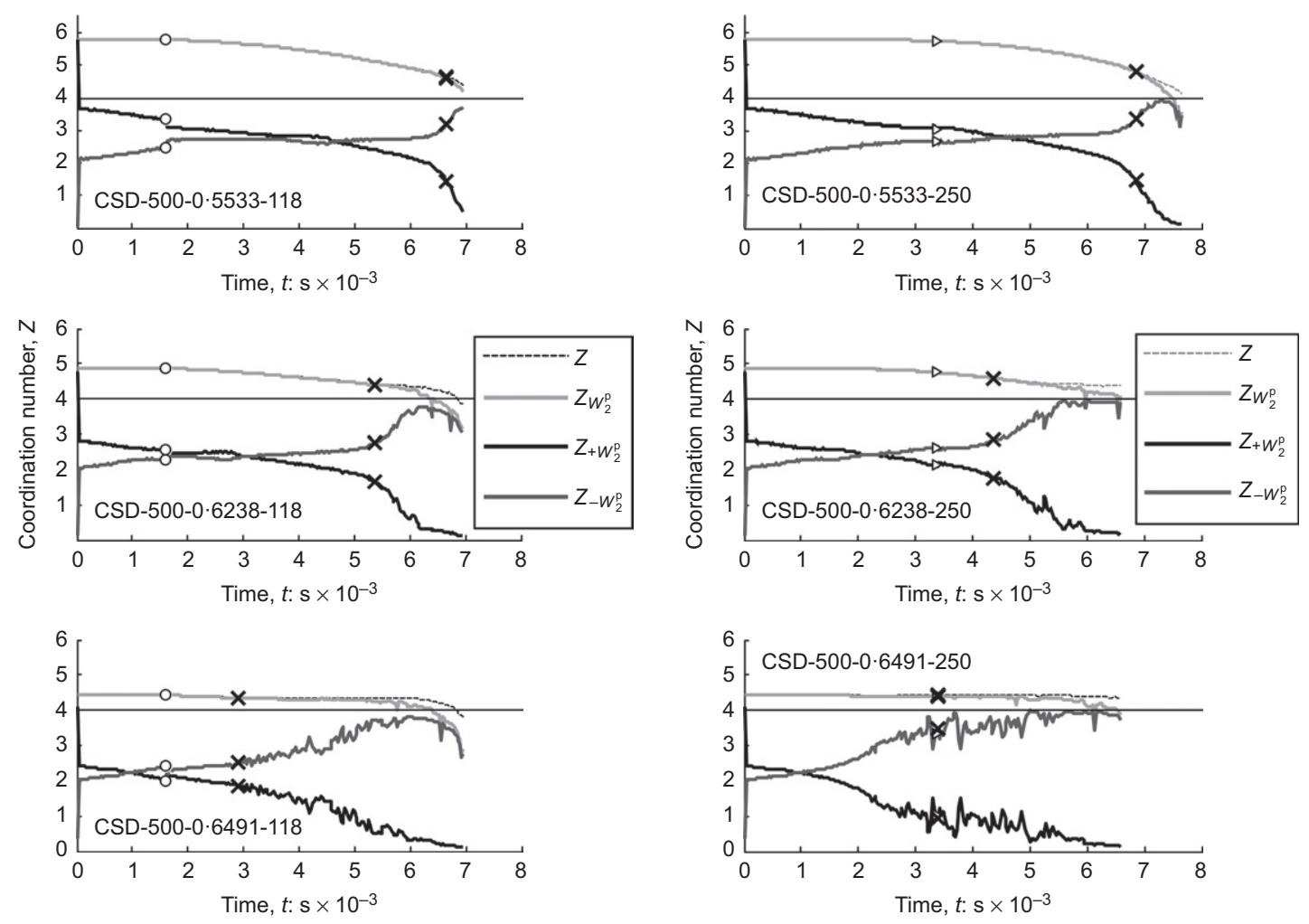

(c)

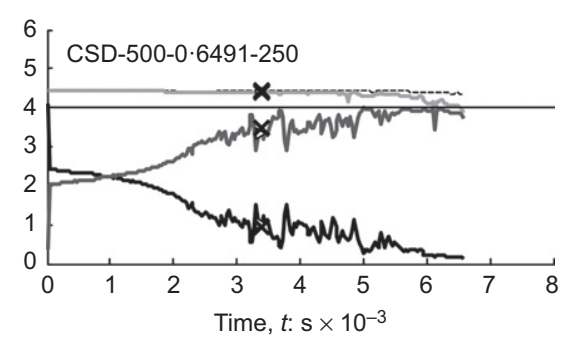

(d)

Fig. 12. (a) Contact anisotropy plotted against time; (b) normal force anisotropy plotted against time; coordination number plotted against time for (c) $q_{\mathrm{csd}}=118 \mathrm{kPa}$ and (d) $q_{\mathrm{csd}}=250 \mathrm{kPa}$. Circle and triangle markers indicate the onset of CSD conditions for $q_{\mathrm{CSD}}=118 \mathrm{kPa}$ and $q_{\mathrm{CSD}}=250 \mathrm{kPa}$, respectively. Cross markers indicate the onset of instability

direction. From the onset of instability onwards, $q$ is transmitted mostly by contacts that are performing a negative $W_{2}^{\mathrm{p}}$, which provokes the overall instability of the system.

An inspection of the micro-mechanical response from CDCV and CSD tests is shown in Fig. 15. Fig. 15(a) shows the increasing evolution of $a_{\mathrm{c}}$ regardless of the loading conditions imposed. However, the shape of increase is different for the CDCV and CSD tests, with the CSD showing an exponential increase. For the CDCV test, the increase in $a_{\mathrm{c}}$ is more pronounced as the initial packing density increases. $a_{\mathrm{c}}$ is 


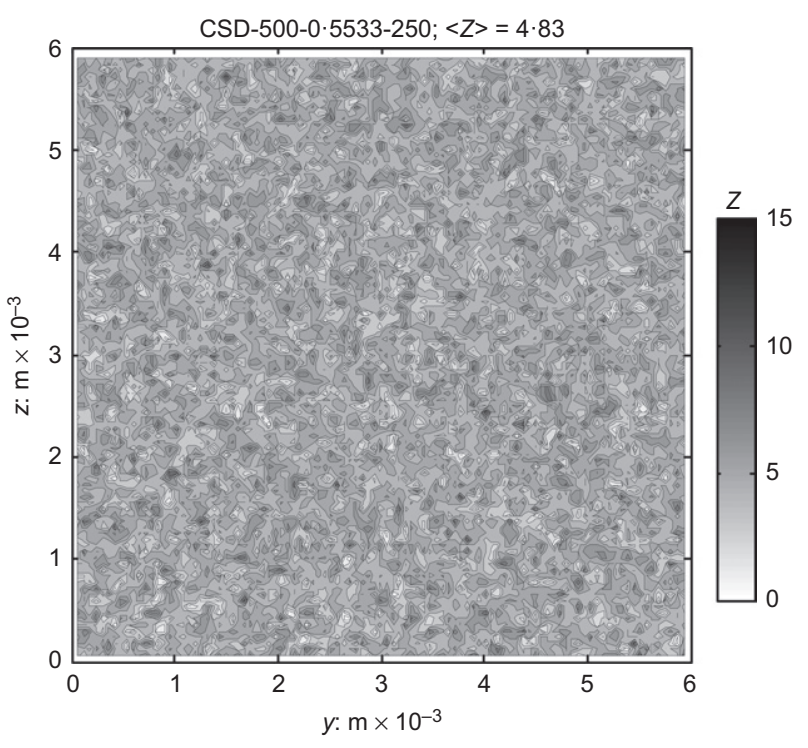

(a)

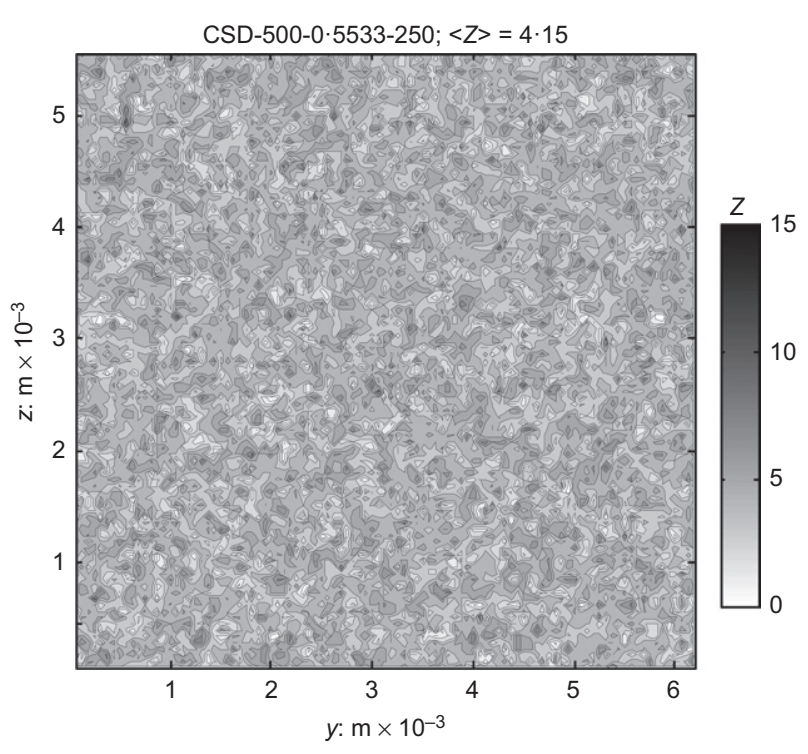

(b)

Fig. 13. Contour plots of coordination number across the plane $y-z$ for the dense sample CSD-500-0·5533-250. (a) At onset of instability with a mean coordination number $(<Z>)$ of 4.83 and (b) at end of test with $\langle Z\rangle=4 \cdot 15$

seen to be higher for the CSD tests at or shortly after their onset of instability than for the CDCV tests. For the loose sample sheared under CDCV, at the onset of instability $a_{\mathrm{c}}$ has not changed significantly. When the sample is liquefying there is a sudden increase of $a_{\mathrm{c}}$, which attains higher values than those at the end of the CSD test. The normal contact force anisotropy $a_{\mathrm{n}}$ is presented in Fig. 15(b). Similarly to $a_{\mathrm{c}}$, there is an increase of $a_{\mathrm{n}}$ regardless of the loading conditions imposed. However, the CSD tests increase exponentially, which does not occur in the CVCD tests. The rate of increase of $a_{\mathrm{n}}$ is also affected by $e_{0}$, with denser samples showing sharper increases than loose ones. For the dense and medium dense samples sheared under CSD conditions, $a_{\mathrm{n}}$ shows lower values than those seen in the CDCV tests. A fairly similar response of $a_{\mathrm{n}}$ is observed for the loose sample, where slightly higher values of $a_{\mathrm{n}}$ are observed for the CSD test, especially at the onset of instability. While $a_{\mathrm{n}}$ at the end of the CSD test shows a sudden drop regardless of initial packing density, $a_{\mathrm{n}}$ for the loose sample sheared under CV conditions increases sharply as liquefaction is taking place within the sample.

$Z$ is plotted against time in Fig. 15(c), where a decay of $Z$ is observed for both CDCV and CSD tests. However, the shape of decay is different for each type of test. $Z$ at the onset of instability for the dense and medium dense samples sheared under CSD is lower than those achieved for the CDCV test. For the loose sample, $Z$ is fairly similar at the onset of instability regardless of the loading conditions imposed. By the end of the test of the loose sample under CDCV conditions, the majority of contacts have been lost as it liquefies. However, as $q$ is still available for the CSD at the end of test, only a small drop in $Z$ is observed.

Radjai et al. (1998) described weak contacts as those that contribute negatively to the deviatoric stress. Following Huang et al. (2016), the characteristic normal contact force $\left(f^{*}\right)$ that marks the transition from negative to positive contribution to the overall deviatoric stress was found at each step, and thus the weak contact proportion was obtained throughout the tests. The weak contact proportion is included in Fig. 15(d); for the dense and medium dense tests a change in the response is observed as soon as either CSD or CV conditions are imposed, with tests under CSD conditions showing an overall lower weak contact proportion than CDCV. For the loose sample, no clear difference in the weak contact proportion can be appreciated after the change in loading conditions. Regardless of initial packing density, tests under CSD conditions show an abrupt decrease in the proportion of weak contacts once the onset of instability is reached. For the case of the loose CDCV test no difference in the proportion of contacts that are weak is seen at the onset of instability; however, at about $7 \mathrm{~ms}$, the CDCV test starts presenting an increase in the weak contact proportion that goes up to 1 when the system has fully liquefied.

\section{CONCLUSIONS}

A series of CSD tests with different densities and stress states were simulated. These simulations were supplemented by $\mathrm{CV}$ simulations, carried out to obtain the relationship between the state parameter and stress ratio at the onset of instability. The simulation schedule included CDCV tests in order to compare the responses under CSD and CV loading conditions. Following Darve et al. (2004) and Hadda et al. (2013), the particle-scale second-order work was employed to identify the onset of instability for the CSD tests.

The macro-response agrees qualitatively with all characteristics of the onset of stability reported by previous published laboratory tests, for example, an increase in strain rate and the loss of controllability to final collapse. For loose states, it was verified that CSD, CV and CDCV tests have the same bifurcation criterion according to the second-order work. The dense sample was found not to agree strictly with the macro-scale second-order work criterion where the second-order work remained positive before and after the onset of instability. However, the loose samples both at CSD and CDCV loading conditions were seen to agree with the macro-scale second-order work, becoming unstable at a minimum in volumetric strain and at the peak of $q$, respectively. The second-order work from a particle scale was seen to be useful in capturing the onset of instability for CSD tests that do not attain an extremum in volumetric strain. Regardless of initial density and deviatoric stress, all samples experienced an onset of instability during the CSD tests. 


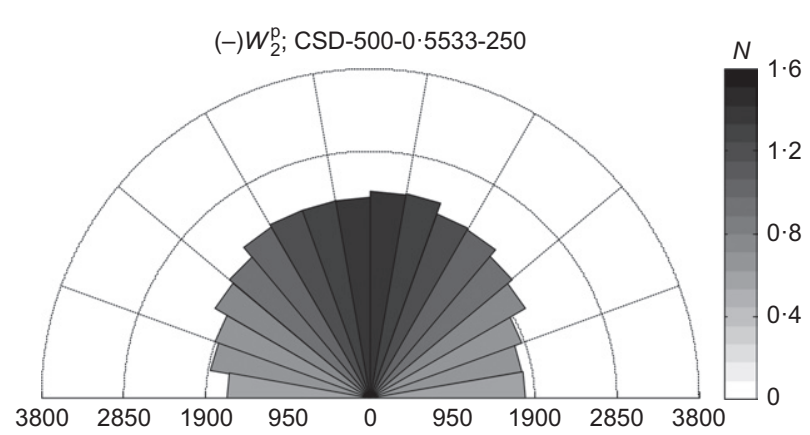

(a)

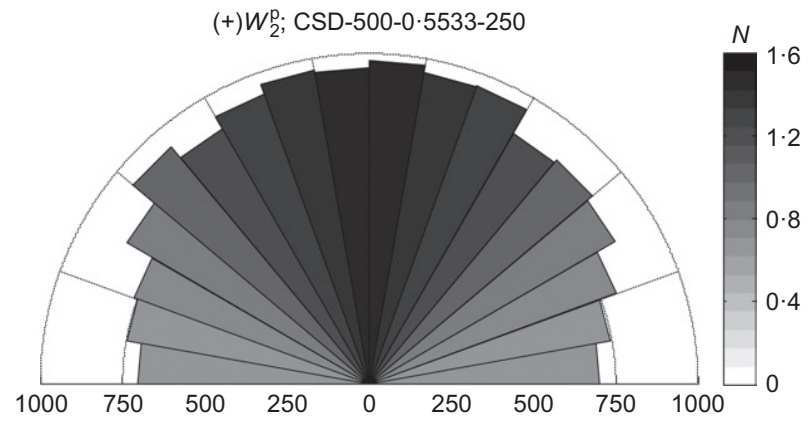

(b)

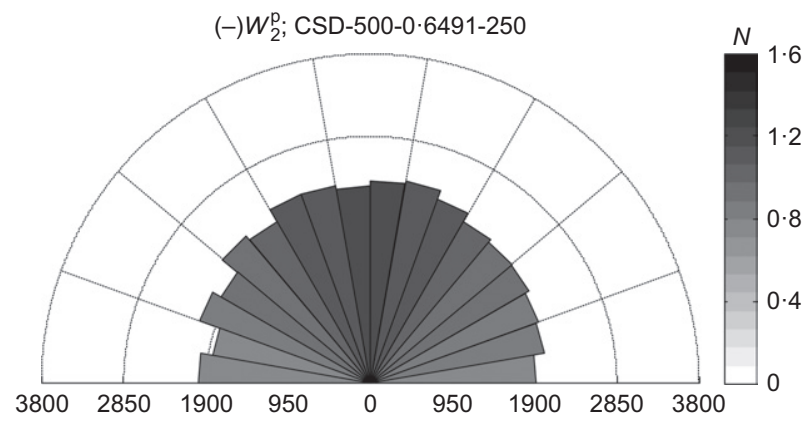

(c)

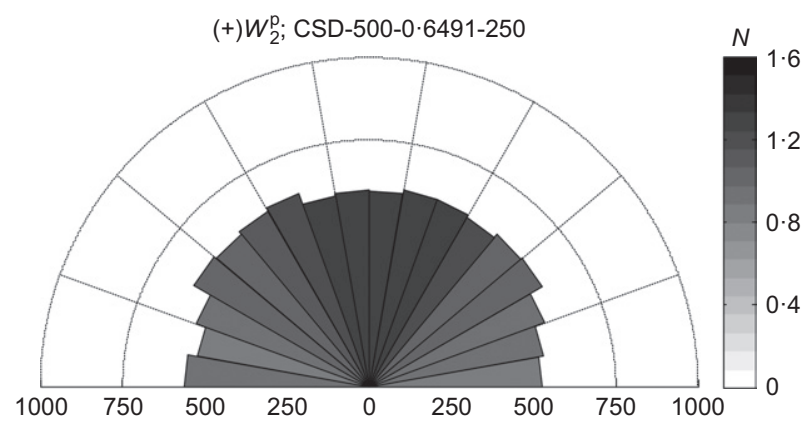

(d)

Fig. 14. Rose diagrams for: (a) contacts carrying negative secondorder work in a dense state; (b) contacts carrying positive second-order work in a dense state; (c) contacts carrying negative secondorder work in a loose state; and (d) contacts carrying positive second-order work in a loose state

By contrast, only the loose sample liquefied under CDCV conditions, with the medium dense and dense sample showing no signs of instability. At the onset of instability a larger proportion of contacts with negative $W_{2}^{p}$ was present in all CSD tests, and these were seen to carry most of the normal contact forces. When the stress ratio at the onset of instability $(\eta)$ was plotted against the initial state parameter $(\psi)$, a linear relationship was found for the CSD tests in $\eta-\psi_{0}$ space, which differs from the $\eta-\psi$ relationship for the $\mathrm{CV}$ tests. Although the $\eta-\psi$ relationship for the $\mathrm{CV}$ tests is useful in determining the conditions of instability for medium dense and loose samples under CSD conditions, it fails to capture the conditions of instability for dense samples under CSD conditions.

The micro-mechanical quantities explored were found to be dependent on loading conditions, and had marked differences before and after the onset of instability. While the structural anisotropy kept increasing regardless of a constant or decaying $q$, the normal contact force anisotropy was seen to decrease once the onset of instability was reached, which happened together with the decay in $q$. The overall stress response post the onset of instability was seen to be a consequence of the interplay between $a_{\mathrm{c}}$ and $a_{\mathrm{n}}$. The micro-mechanical responses of the CSD tests differed from those of the CDCV tests, with CSD tests presenting higher geometrical and mechanical anisotropies at the onset of instability compared to CDCV tests. The rate of change in all micro-quantities studied was not affected by the onset of instability for the CDCV tests and it was only at liquefaction that a sudden change was observed. Although all samples sheared under CSD conditions showed unstable behaviour, only the loose sample liquefied under CDCV conditions, with the medium dense and dense samples showing no signs of instabilities. Therefore, it is important to note that the constant shear drained loading conditions can result in more unfavourable situations than for undrained loading conditions.

\section{ACKNOWLEDGEMENTS}

This study was supported by The University of Hong Kong SPACE research fund and the Research Grants Council of Hong Kong - General Research Fund (grant no. 17203614). This research is conducted using the HKU Information Technology Services research computing facilities that are supported in part by the Hong Kong UGC special equipment grant (SEG HKU09).

\author{
NOTATION \\ $a_{\mathrm{n}}$ normal contact force anisotropy \\ e void ratio \\ $\boldsymbol{F}_{i j}^{\mathrm{n}} \quad$ average normal contact force tensor \\ $\overline{f_{\mathrm{n}}}(\Omega)$ probability distribution function of the average normal \\ contact force tensor \\ $f^{*}$ characteristic normalised normal contact force \\ $G$ particle shear modulus \\ $I$ inertial number \\ $k$ contact stiffness \\ $l_{i}^{\mathrm{c}} \quad$ branch vector \\ $m$ particle mass \\ $N_{\mathrm{c}} \quad$ total number of contacts \\ $p^{\prime}$ mean effective stress \\ $p_{0}^{\prime}$ mean effective stress after isotropic compression \\ $q$ deviatoric stress \\ $V \quad$ volume of periodic cell \\ $W_{2}^{\mathrm{p}} ; \mathrm{d}^{2} W \quad$ second-order work at a particle scale; second-order \\ work at a macro scale \\ $Z$ coordination number \\ $\varepsilon_{1} \quad$ major principal strain \\ $\varepsilon_{\mathrm{v}} \quad$ volumetric strain \\ $\eta \quad$ stress ratio $\left(=q / p^{\prime}\right)$ \\ $\mu$ inter-particle friction coefficient \\ $v$ particle Poisson ratio \\ $\rho$ particle density \\ $\overline{\boldsymbol{\sigma}}_{i j}$ stress tensor \\ $\boldsymbol{\Phi}_{i j}$ fabric tensor
}

$\Phi_{1} ; \Phi_{2} ; \Phi_{3}$ major, intermediate and minor eigenvalues of the fabric tensor $\left(\boldsymbol{\Phi}_{i j}\right)$

$\psi \quad$ state parameter $\left(=e_{0}-e_{\mathrm{cs}}\right)$ 

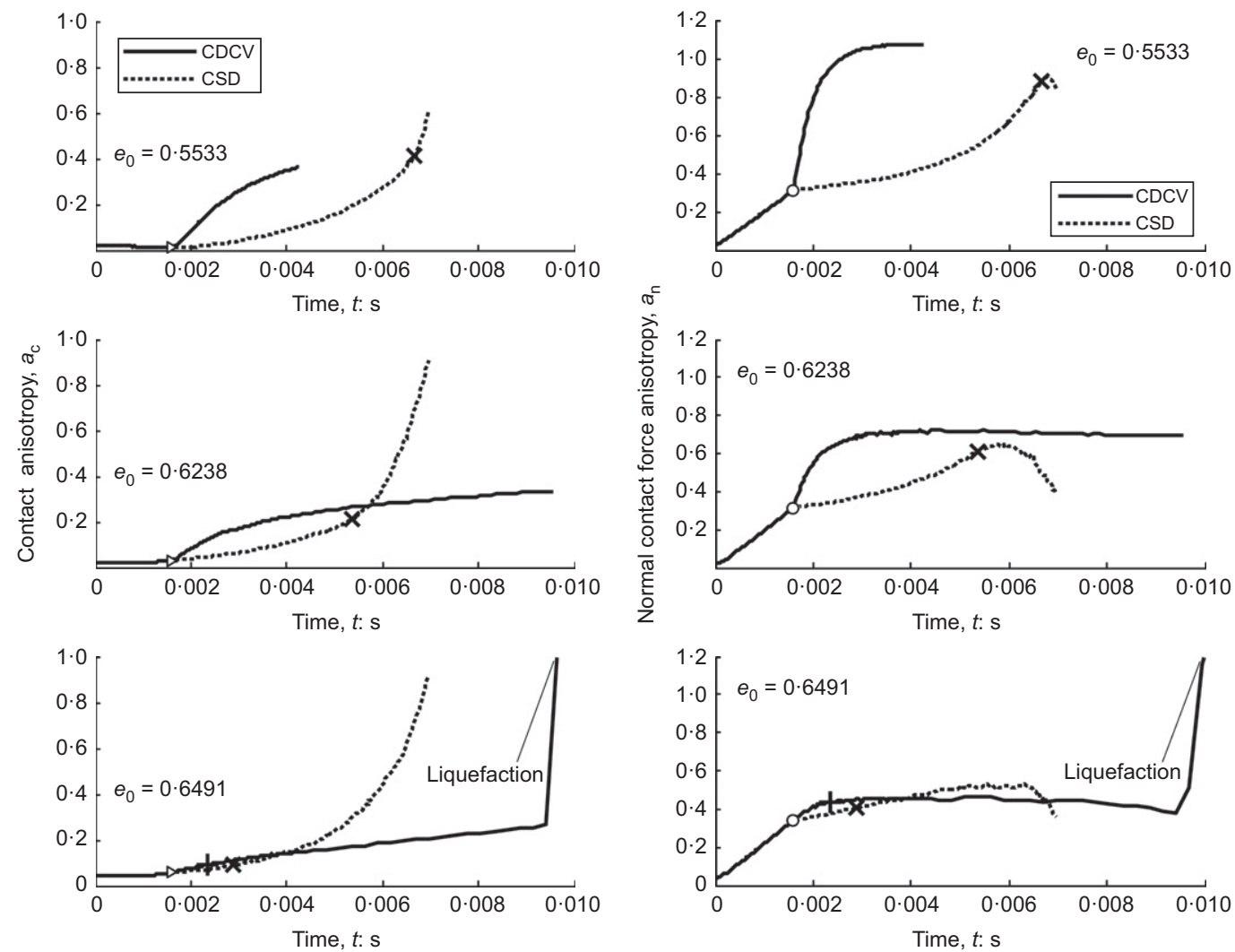

(a)
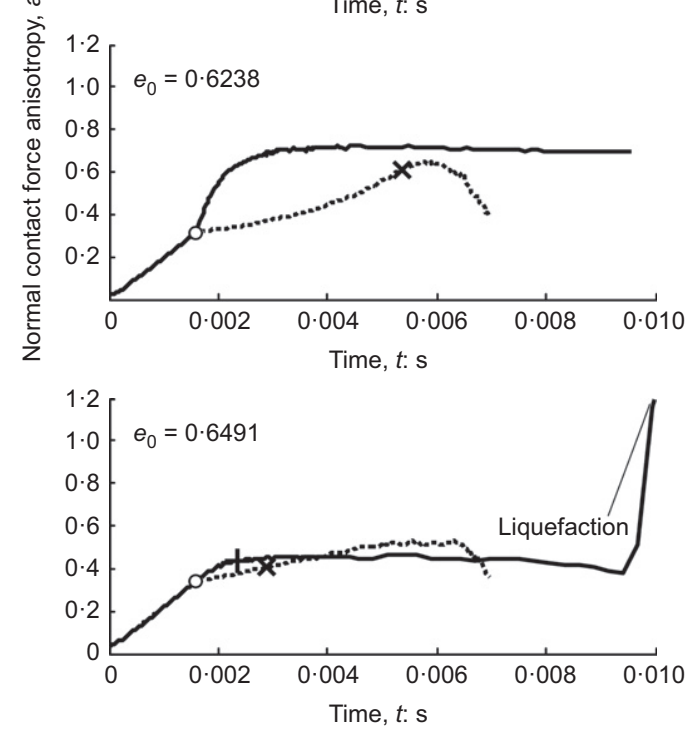

(b)
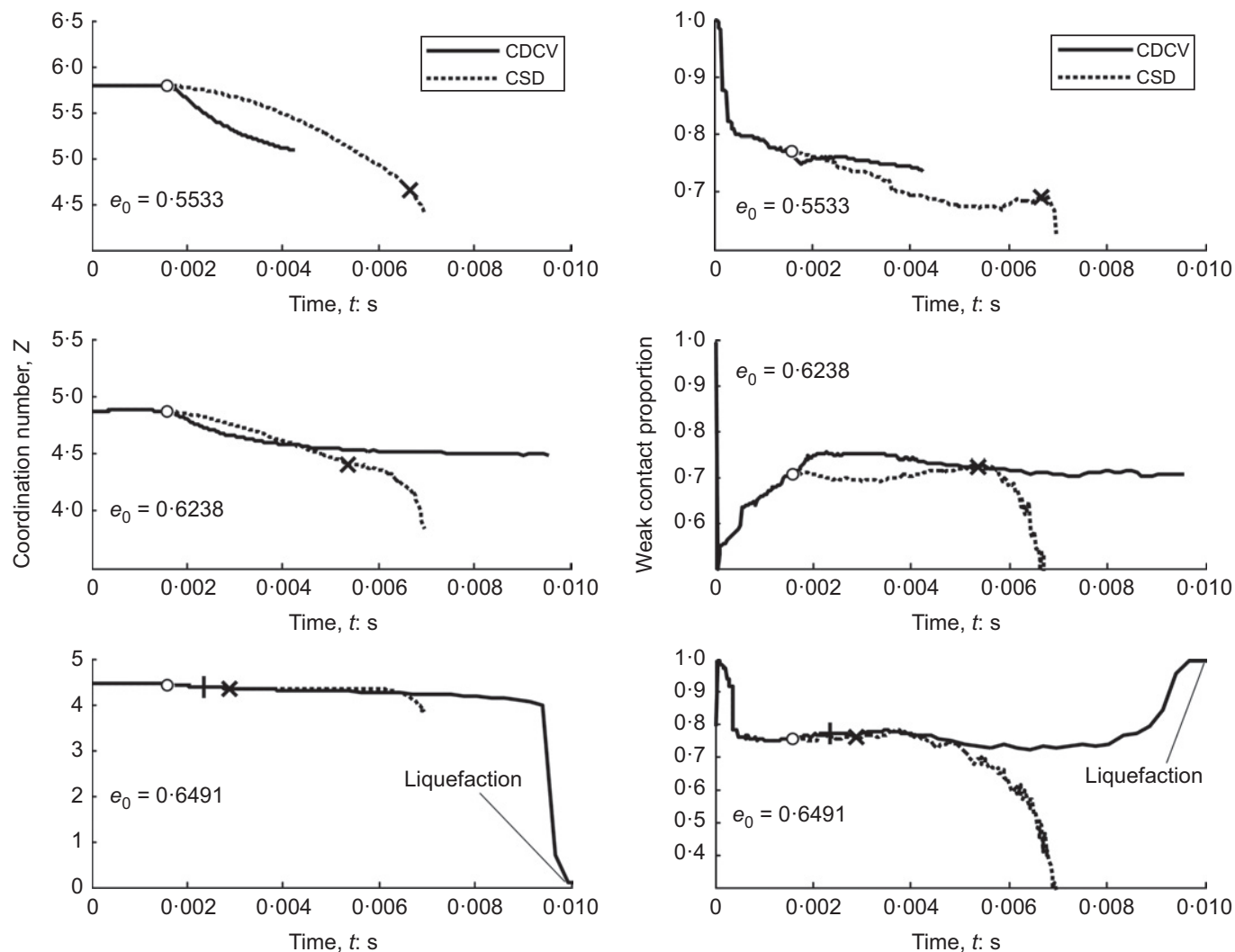

(c)

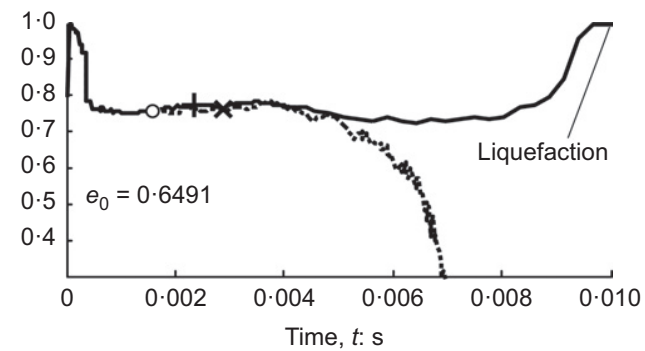

(d)

Fig. 15. Comparison of the micro response plotted against time for CSD and CDCV tests: (a) contact anisotropy, $a_{\mathrm{c}}$; (b) normal contact force anisotropy, $a_{n}$; (c) coordination number; (d) weak contact proportion. Cross markers indicate the onset of instability for CSD tests and plus sign markers indicate the onset of instability for the CDCV test 


\section{REFERENCES}

Anderson, S. A. \& Riemer, M. F. (1995). Collapse of saturated soil due to reduction in confinement. J. Geotech. Engng 121, No. 2, 216-219.

Bagi, K. (1996). Stress and strain in granular assemblies. Mech. Mater. 22, No. 3, 165-177.

Been, K. \& Jefferies, M. G. (1985). A state parameter for sands. Géotechnique 35, No. 2, 99-112, http://dx.doi.org/10.1680/geot. 1985.35.2.99.

Chu, J. \& Leong, W. K. (2001). Pre-failure strain softening and pre-failure instability of sand: a comparative study. Géotechnique 51, No. 4, 311-321, http://dx.doi.org/10.1680/ geot.2001.51.4.311.

Chu, J., Leroueil, S. \& Leong, W. K. (2003). Unstable behaviour of sand and its implication for slope instability. Can. Geotech. J. 40, No. 5, 873-885.

Chu, J., Leong, W. K., Loke, W. L. \& Wanatowski, D. (2012). Instability of loose sand under drained conditions. J. Geotech. Engng 138, No. 2, 207-216.

Cundall, P. A. \& Strack, O. D. L. (1979). A discrete numerical model for granular assemblies. Géotechnique 29, No. 1, 47-65, http:// dx.doi.org/10.1680/geot.1979.29.1.47.

da Cruz, F., Emam, S., Prochnow, M., Roux, J. N. \& Chevoir, F. (2005). Rheophysics of dense granular materials: discrete simulation of plane shear flows. Phys. Rev. E 72, No. 2, 021309.

Daouadji, A., AlGali, H., Darve, F. \& Zeghloul, A. (2010). Instability of granular materials: experimental evidence of diffuse mode of failure for loose sands. J. Engng Mech. 136, No. 5, 575-588.

Darve, F. \& Roguiez, X. (1998). Homogeneous bifurcation in soils. In Localization and bifurcation theory for soils and rocks (eds A. Thoshihisa, F. Oka and A. Yashima), pp. 43-50. Rotterdam, the Netherlands: Balkema.

Darve, F., Servant, G., Laouafa, F. \& Khoa, H. D. V. (2004). Failure in geomaterials: continuous and discrete analyses. Comput. Methods Appl. Mech. Engng 193, No. 27-29, 3057-3085.

Darve, F., Sibille, L., Daouadji, A. \& Nicot, F. (2007). Bifurcations in granular media: macro- and micro-mechanics approaches. Comptes Rendus Mécanique 335, No. 9-10, 496-515.

Eckersley, J. D. (1990). Instrumented laboratory flowslides. Géotechnique 40, No. 3, 489-502, http://dx.doi.org/10.1680/ geot.1990.40.3.489.

Gajo, A., Piffer, L. \& de Polo, F. (2000). Analysis of certain factors affecting the unstable behaviour of saturated loose sand. Mech. Cohesive-Frictional Mater. 5, No. 3, 215-237.

Guo, N. \& Zhao, J. (2013). The signature of shear-induced anisotropy in granular media. Comput. Geotech. 47, 1-15.

Hadda, N., Nicot, F., Bourrier, F., Sibille, L., Radjai, F. \& Darve, F. (2013). Micromechanical analysis of second order work in granular media. Granular Matter 15, No. 2, 221-235.

Hill, R. (1958). A general theory of uniqueness and stability in elastic- plastic solids. J. Mech. Phys. Solids 6, No. 3, 236-249.

Huang, X., Hanley, K. J., O’Sullivan, C. \& Kwok, C.Y. (2014). Effect of sample size on the response of DEM samples with a realistic grading. Particuology 15, 107-115.

Huang, X., O’Sullivan, C., Hanley, K. J. \& Kwok, C.Y. (2016). Partition of the contact force network obtained in discrete element simulations of element tests. Comput. Particle Mech., http://dx.doi/org/10.1007/s40571-015-0095-y.

Jop, P., Forterre, Y. \& Pouliquen, O. (2006). A constitutive law for dense granular flows. Nature 441, No. 8, 727-730.

Li, X. S. \& Wang, Y. (1998). Linear representation of steady-state line for sand. J. Geotech. Geoenviron. Engng 124, No. 12, 1215-1217.

Lindenberg, J. \& Koning, H. L. (1981). Critical density of sand. Géotechnique 31, No. 2, 231-245, http://dx.doi.org/10.1680/ geot.1981.31.2.231.
Lopera, P. J. C., Kwok, C.Y., O’Sullivan, C., Huang, X. \& Hanley, K. J. (2016). Assessing the quasi-steady conditions for shearing in granular media within the critical state soil mechanics framework. Soils Found. 56, No. 1, 152-159.

MiDi, G. D. R. (2004). On dense granular flows. Eur. Phys. J. E 14, No. 4, 341-365.

Nicot, F., Daouadji, A., Laouafa, F. \& Darve, F. (2011). Second-order work, kinetic energy and diffuse failure in granular materials. Granular Matter 13, No. 1, 19-28.

Ning, Z., Evans, M. T. \& Andrade, J. (2013). Particulate study of drained diffuse instability in granular material. In Proceedings of ASCE geocongress 2013: stability and performance of slopes and embankments III, San Diego, CA (eds C. Meehan, D. Pradel, M. A. Pando and J. F. Labuz), Geotechnical Special Publication No. 231, pp. 1290-1299. Reston, VA, USA: American Society of Civil Engineers.

Nova, R. (1994). Controllability of the incremental response of soil specimens subjected to arbitrary loading programs. J. Mech. Behaviour Mater. 5, No. 2, 193-201.

Olson, S. M., Stark, T. D., Walton, W. H. \& Castro, G. (2000). 1907 static liquefaction flow failure of the north dike of Wachusett dam. J. Geotech. Geoenviron. Engng 126, No. 12, 1184-1193.

Plimpton, S. (1995). Fast parallel algorithms for short-range molecular dynamics. J. Comput. Phys. 117, No. 1, 1-19.

Potyondy, D. O. \& Cundall, P. A. (2004). A bonded-particle model for rock. Int. J. Rock Mech. Min. 41, No. 8, 1329-1364.

Radjai, F., Wolf, D. E., Jean, M. \& Moreau, J. (1998). Bimodal character of stress transmission in granular packings. Phys. Rev. Lett. 80, No. 1, 61-64.

Ramos, A. M., Andrade, J. E. \& Lizcano, A. (2012). Modelling diffuse instabilities in sands under drained conditions. Géotechnique 62, No. 6, 471-478, http://dx.doi.org/10.1680/ geot.10.P.109.

Rothenburg, L. \& Bathurst, R. J. (1989). Analytical study of induced anisotropy in idealized granular materials. Géotechnique 39, No. 4, 601-614, http://dx.doi.org/10.1680/ geot.1989.39.4.601.

Sasitharan, S., Robertson, P. K., Sego, D. C. \& Morgenstern, N. R. (1993). Collapse behavior of sand. Can. Geotech. J. 30, No. 4, 569-577.

Satake, M. (1982). Fabric tensor in granular materials. In Deformation and failure of granular materials (eds P. A. Vermeer and H. J. Luger), pp. 63-68. Rotterdam, the Netherlands: Balkema.

Sawicki, A. \& Swidzinski, W. (2010). Modelling of pre-failure instabilities of sand. Comput. Geotech. 37, No. 6, 781-788.

Senetakis, K., Coop, M. R. \& Todisco, M. C. (2013). Tangential load-deflection behaviour at the contacts of soil particles. Géotechnique Lett. 3, No. 2, 59-66.

Sibille, L., Nicot, F., Donze, F. \& Darve, F. (2007). Material instability in granular assemblies from fundamentally different models. Int. J. Numer. Analyt. Methods Geomech. 31, No. 3, 457-481.

Skopek, P., Morgenstern, N. R., Robertson, P. K. \& Sego, D. C. (1994). Collapse of dry sand. Can. Geotech. J. 31, No. 6, 1003-1008.

Sladen, J. A., D'Hollander, R. D. \& Krahn, J. (1985). The liquefaction of sands, a collapse surface approach. Can. Geotech. J. 22, No. 4, 564-578.

Thornton, C. (2000). Numerical simulations of deviatoric shear deformation of granular media. Géotechnique 50, No. 1, 43-53, http://dx.doi.org/10.1680/geot.2000.50.1.43.

Zhu, J. H. \& Anderson, S. A. (1998). Determination of shear strength of Hawaiian residual soil subjected to rainfall-induced landslides. Géotechnique 48, No. 1, 73-82, http://dx.doi.org/ 10.1680/geot.1998.48.1.73. 